03

\title{
Элементарный излучатель, расположенный на границе или внутри слоистой структуры
}

\author{
(C) А.Б. Петрин \\ Объединенный институт высоких температур РАН, \\ 125412 Москва, Россия \\ e-mail: a_petrin@mail.ru \\ Поступила в редакцию 04.06.2020 г. \\ В окончательной редакции 04.06.2020 г. \\ Принята к публикации 03.07.2020 г.
}

\begin{abstract}
Рассмотрена строгая теория излучения элементарного диполя, расположенного на границе или внутри плоскослоистой структуры. Для конкретного случая излучения диполя, расположенного на свободной границе одной пленки, продемонстрирован метод аналитического упрощения решения. Этот метод позволил привести формулы для излучаемых полей к одномерным интегралам, что существенно упростило анализ задачи и ускорило численные расчеты. В качестве конкретного технического приложения теории были получены диаграммы направленности точечных излучателей (молекул, наноструктур), расположенных на свободной поверхности металлической пленки в схеме Кречмана и имеющих индуцированный дипольный момент вдоль поверхности пленки. Определено влияние поверхностной волны на направленные свойства излучателей.
\end{abstract}

Ключевые слова: нанофокусировка, поверхностные плазмоны, оптические сенсоры.

DOI: $10.21883 /$ OS.2020.11.50171.168-20

\section{Введение}

В последнее время значительный интерес привлекают физические явления, которые сопровождают возбуждение поверхностной плазмонной волны на поверхности металлической пленки по схеме Кречмана [1] (рис. 1). Поверхностная плазмонная волна распространяется вдоль поверхности металла и локализуется вблизи его поверхности [2,3], поэтому даже ничтожные изменения показателя преломления в приповерхностной области сильно влияют на характер ее распространения. На этом основывается широкое использование схемы Кречмана в различного рода высокочувствительных датчиках, реагирующих на изменение показателя преломления тонкого (олиго- или моно-молекулярного) поверхностного слоя [4].

Как показали исследования [5], амплитуда поверхностной волны на свободной границе пленки в схеме Кречмана более чем на порядок выше амплитуды падающей волны. Так как в свободном пространстве над пленкой не возбуждаются распространяющиеся волны, это дает возможность, помещая на свободную поверхность наночастицы или отдельные молекулы, наблюдать именно их излучение, направленное в сторону свободного полупространства. Это излучение порождается наведенными электрическими дипольными моментами наночастиц, которые индуцируются поверхностной волной. Важно, что только точечные объекты будут излучать распространяющиеся волны в свободное пространство, и исключительно это излучение можно будет наблюдать с помощью микроскопа, при этом можно быть полностью уверенным, что наблюдаемый в микроскоп свет исходит именно из точечных источников на поверхности. В связи с этим возникает фундаментальный вопрос о том, как влияет металлическая пленка (или в общем случае многопленочная структура) в схеме Кречмана на пространственное излучение в свободное пространство над пленкой от точечного излучателя, расположенного на поверхности.

Теоретические методы нахождения излучения от элементарного точечного электрического диполя, расположенного на плоской границе двух сред, были развиты на заре эры использования электромагнитных волн для передачи сигналов вдоль земной поверхности [6]. Эти методы получили дальнейшее развитие в связи с воз-

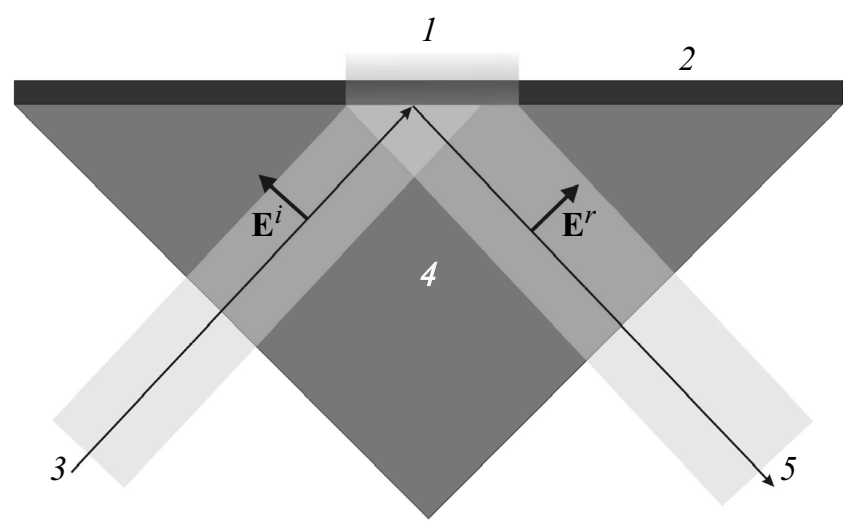

Рис. 1. Возбуждение поверхностной плазмонной волны 1 на поверхности металлической пленки 2 по схеме Кречмана. Падающая поляризованная волна 3 со стороны призмы 4 порождает на свободной границе поверхностную плазмонную 1 и отраженную 5 волны. 
никающими новыми задачами радиосвязи $[7,8]$. Развитие антенной техники привело к развитию теории излучения элементарного диполя, расположенного на границе плоскослоистой среды и теории излучения микрополосковых антенн [9-11], которые являются наилучшими конструкциями с точки зрения их совместимости с микрополосковыми интегральными схемами и которые играют особую роль в современной технике, так как могут быть изготовлены из фольгированных материалов высокопроизводительными методами печати и травления.

Существующие теоретические методы расчета излучения антенн, расположенных на границе слоистых структур, сложны (описываются в терминах диадных функций Грина [12]). Кроме того, сама теория распространения волн в слоистых структурах содержит много тонких моментов, таких как необходимость правильного выбора ветвей аналитических функций при записи волн в слоях, которые могут приводить к чудесам вроде суперразрешения [13]. Поэтому в настоящей работе предложен вариант строгой электромагнитной теории излучения элементарного диполя, расположенного на границе или внутри плоскослоистой структуры. Для случая излучения диполя, расположенного на свободной границе одной пленки, ниже продемонстрирован метод аналитического упрощения решения, имеющий, как кажется, потенциально общетеоретическое значение. Этот метод позволил привести формулы для излучаемых полей к одномерным интегралам, что существенно упростило анализ задачи и ускорило численные расчеты.

\section{Постановка задачи. Излучение элементарного излучателя, расположенного внутри плоскослоистой структуры}

Рассмотрим задачу излучения электромагнитной волны точечным источником тока единичной амплитуды, изменяющимся во времени гармонически с циклической частотой $\omega$. Пусть этот источник расположен в плоской слоистой структуре, состоящей из нескольких пленок, и из окружающих слоистую структуру двух полупространств. Для определенности сначала будем считать, что источник расположен в одной из пленок, а затем обобщим эту задачу на случай, когда источник расположен на границе между слоями.

Пусть общее число пленок равно $N_{f}$, толщина $m$-й пленки равна $d_{m}$ и полная толщина слоистой структуры равна $d_{\text {tot }}=\sum_{m=1}^{N_{f}} d_{m}$. Общее число границ между пленками обозначим как $N=N_{f}+1$. Пронумеруем области пространства $j=1, \ldots,(N+1)$ (на рис. 2 показана для примера задача с $\mathrm{N}=4$ и $\left.N_{f}=3\right)$. Предположим, что пленки имеют абсолютные комплексные диэлектрические и магнитные проницаемости, равные $\varepsilon_{j}$ и $\mu_{j}$ на рассматриваемой частоте $\omega$, а перед и за слоистой

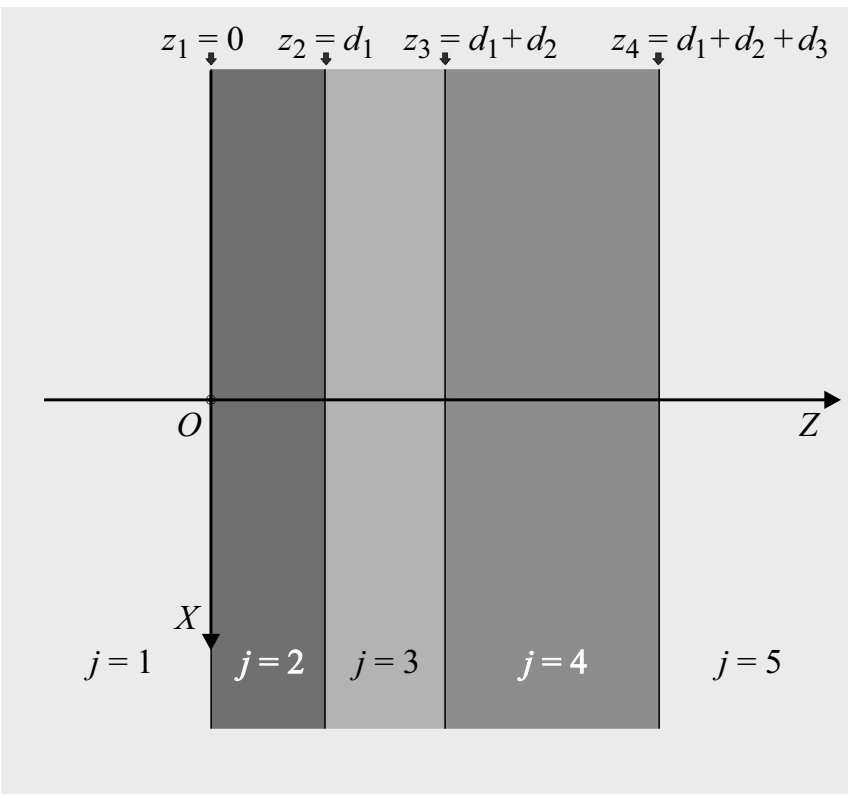

Рис. 2. Геометрия плоскослоистой структуры, состоящей из трех пленок.

структурой находятся однородные полупространства с проницаемостями $\varepsilon_{1}, \mu_{1}$ и $\varepsilon_{N+1}, \mu_{N+1}$ (свободное пространство). Обозначим также через $z_{j}$ координаты $N$ границ пленок по оси $Z$ следующим образом: $z_{1}=0$, $z_{j}=\sum_{m=1}^{j-1} d_{m}$ при $j=2, \ldots, N$.

Уравнения Максвелла для области с номером $j$ можно записать в виде

$$
\begin{gathered}
\operatorname{rot} \mathbf{E}_{j}=i \omega \mathbf{B}_{j}, \\
\operatorname{rot} \mathbf{B}_{j}=\mu_{j}\left(-i \omega \varepsilon_{j} \mathbf{E}_{j}+\mathbf{J}_{j}\right),
\end{gathered}
$$

где $\mathbf{E}_{j}, \mathbf{B}_{j}$ и $\mathbf{J}_{j}-$ векторы напряженности электрического поля, индукция магнитного поля и плотность стороннего (известного) тока в области с номером $j$ (если источник тока отсутствует в области $j$, то $\mathbf{J}_{j}=0$ ). Предполагается комплексное временное представление в виде $e^{-i \omega t}$.

Решая уравнения Максвелла в каждой области задачи, найдем электромагнитное поле во всех областях. Рассмотрим сначала следующую вспомогательную задачу.

\section{Распространение электромагнитной волны в слое, свободном от сторонних токов}

Пусть сторонних токов нет в области с номером $j$ между границами $z_{j-1}$ и $z_{j}$ (рис. 3). Диэлектрическая и магнитная проницаемости среды в этой пленке равны $\varepsilon_{j}$ и $\mu_{j}$ соответственно. Тогда, учитывая, что в этой области справедливо уравнение $\operatorname{div} \mathbf{D}_{j}=0$, из уравнений (1) и (2) получаем уравнения для электромагнитных полей 


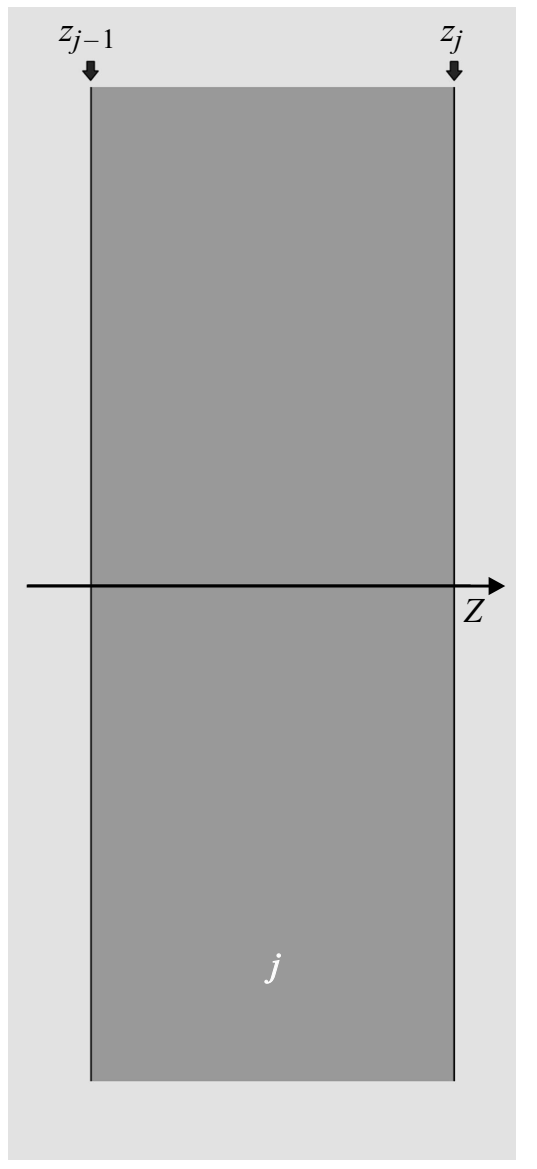

Рис. 3. Пленка с номером $j$, расположенная между границами $z_{j+1}$ и $z_{j}$.

в виде

$$
\begin{aligned}
& \operatorname{rot} \operatorname{rot} \mathbf{E}_{j}-\omega^{2} \varepsilon_{j} \mu_{j} \mathbf{E}_{j}=0, \\
& \operatorname{rot} \operatorname{rot} \mathbf{B}_{j}-\omega^{2} \varepsilon_{j} \mu_{j} \mathbf{B}_{j}=0 .
\end{aligned}
$$

B рассматриваемой области $\operatorname{div} \mathbf{E}_{j}=0 \quad$ и $\operatorname{div} \mathbf{B}_{j}=0$. Учитывая векторное тождество $\operatorname{rot} \operatorname{rot} \mathbf{F}=$ $=\operatorname{grad} \operatorname{div} \mathbf{F}-\Delta \mathbf{F}$, где $\Delta=\partial_{x x}^{2}+\partial_{y y}^{2}+\partial_{z z}^{2}-$ оператор Лапласа, из (3) и (4) получим

$$
\begin{aligned}
\Delta \mathbf{E}_{j}+\omega^{2} \varepsilon_{j} \mu_{j} \mathbf{E}_{j} & =0, \\
\Delta \mathbf{B}_{j}+\omega^{2} \varepsilon_{j} \mu_{j} \mathbf{B}_{j} & =0 .
\end{aligned}
$$

Подставим в полученные выше уравнения компоненты полей в виде фурье-разложений. Представление для $x$ компоненты электрического поля примем в виде

$$
E_{j, x}(x, y, z)=\frac{1}{(2 \pi)^{2}} \int_{-\infty}^{+\infty} \int_{-\infty}^{+\infty} \tilde{E}_{j, x}(\xi, \eta, z) e^{i(\xi x+\eta y)} d \xi d \eta
$$

где фурье-образы определяются выражением

$$
\tilde{E}_{j, x}(\xi, \eta, z)=\int_{-\infty}^{+\infty} \int_{-\infty}^{+\infty} E_{j, x}(x, y, z) e^{-i(\xi x+\eta y)} d x d y .
$$

Для остальных компонент полей будем использовать аналогичные представления и соответствующие символы.

В компонентах уравнения (5) и (6) имеют вид

$$
\partial_{x x}^{2} E_{j, x}+\partial_{y y}^{2} E_{j, x}+\partial_{z z}^{2} E_{j, x}+\omega^{2} \mu_{j} \varepsilon_{j} E_{j, x}=0
$$

и аналогичные уравнения для $E_{j, y}, E_{j, z}$ и составляющих магнитного поля, которые выписывать не будем. Переходя к фурье-образам, получаем из (5) уравнения

$$
\begin{gathered}
\frac{d^{2} \tilde{E}_{j, x}}{d z^{2}}+\gamma_{j}^{2} \tilde{E}_{j, x}=0, \quad \frac{d^{2} \tilde{E}_{j, y}}{d z^{2}}+\gamma_{j}^{2} \tilde{E}_{j, y}=0, \\
\frac{d^{2} \tilde{E}_{j, z}}{d z^{2}}+\gamma_{j}^{2} \tilde{E}_{j, z}=0,
\end{gathered}
$$

где $\gamma_{j}=\sqrt{k_{j}^{2}-\xi^{2}-\eta^{2}}, k_{j}=\omega \sqrt{\mu_{j} \varepsilon_{j}}$.

Решения уравнений (7) для направлений распространения волн вдоль „+““ и против оси $Z$ можно записать в виде

$$
\mathbf{E}_{j}^{ \pm}(x, y, z)=\frac{1}{(2 \pi)^{2}} \int_{-\infty}^{+\infty} \int_{-\infty}^{+\infty}\left(\begin{array}{c}
\hat{E}_{j, x}^{ \pm} \\
\hat{E}_{j, y}^{ \pm} \\
\hat{E}_{j, z}^{ \pm}
\end{array}\right) e^{ \pm i \gamma_{j} z} e^{i(\xi x+\eta y)} d \xi d \eta
$$

В рассматриваемом случае $\operatorname{div} \mathbf{E}_{j}=0$ и, следовательно, $\xi \hat{E}_{j, x}+\eta \hat{E}_{j, y} \pm \gamma \hat{E}_{j, z}=0$. Тогда (8) можно переписать в виде

$$
\begin{aligned}
\mathbf{E}_{j}^{ \pm}(x, y, z)= & \frac{1}{(2 \pi)^{2}} \int_{-\infty}^{+\infty} \int_{-\infty}^{+\infty}\left(\begin{array}{cc}
1 & 0 \\
0 & 1 \\
\mp \xi / \gamma_{j} & \mp \eta / \gamma_{j}
\end{array}\right)\left(\begin{array}{c}
\hat{E}_{j, x}^{ \pm} \\
\hat{E}_{j, y}^{ \pm}
\end{array}\right) \\
& \times e^{ \pm i \gamma_{j} z} e^{i(\xi x+\eta y)} d \xi d \eta .
\end{aligned}
$$

Поэтому общее решение уравнений (7) в области $\left[z_{j-1}, z_{j}\right]$ можно записать в виде

$$
\begin{aligned}
\mathbf{E}_{j}(x, y, z)= & \frac{1}{(2 \pi)^{2}} \int_{-\infty}^{+\infty} \int_{-\infty}^{+\infty}\left(\begin{array}{cc}
1 & 0 \\
0 & 1 \\
-\xi / \gamma_{j} & -\eta / \gamma_{j}
\end{array}\right)\left(\begin{array}{c}
\hat{E}_{j, x}^{+} \\
\hat{E}_{j, y}^{+}
\end{array}\right) \\
& \times e^{i \gamma_{j}\left(z-z_{j-1}\right)} e^{i(\xi x+\eta y)} d \xi d \eta \\
& +\frac{1}{(2 \pi)^{2}} \int_{-\infty}^{+\infty} \int_{-\infty}^{+\infty}\left(\begin{array}{cc}
1 & 0 \\
0 & 1 \\
\xi / \gamma_{j} & \eta / \gamma_{j}
\end{array}\right)\left(\begin{array}{c}
\hat{E}_{j, x}^{-} \\
\hat{E}_{j, y}^{-}
\end{array}\right) \\
& \times e^{-i \gamma_{j}\left(z-z_{j}\right)} e^{i(\xi x+\eta y)} d \xi d \eta .
\end{aligned}
$$

Обратим внимание на форму записи волн. Они распространяются со стороны границ. При этом подразумевается правильный выбор ветвей аналитических функций $\gamma_{j}$, который обеспечивает сходимость интегралов (в 
выражение для $\gamma_{j}$ входит двузначная функция - корень квадратный). В случае неправильного выбора экспоненциально усиливаются гармоники с малыми волновыми числами, что приводит к нефизичным результатам. Подробности о выборе правильных аналитических ветвей можно найти в $[8,14]$.

Из уравнения $\operatorname{rot} \mathbf{E}_{j}=i \omega \mathbf{B}_{j}$ найдем $x$ - и $y$-компоненты магнитного поля. Для соответствующих фурьекомпонент найдем

$$
\begin{aligned}
\hat{\mathbf{B}}_{j}^{+}= & \mathbf{e}_{x}\left(\frac{\eta}{\omega} \hat{E}_{j, z}^{+}-\frac{\gamma_{j}}{\omega} \hat{E}_{j, y}^{+}\right)-\mathbf{e}_{y}\left(\frac{\xi}{\omega} \hat{E}_{j, z}^{+}-\frac{\gamma_{j}}{\omega} \hat{E}_{j, x}^{+}\right) \\
& +\mathbf{e}_{z}\left(\frac{\xi}{\omega} \hat{E}_{j, y}^{+}-\frac{\eta}{\omega} \hat{E}_{j, x}^{+}\right),
\end{aligned}
$$

или, учитывая $\operatorname{div} \mathbf{E}_{j}=0$, подставим $\hat{E}_{j, z}^{+}=-\xi \hat{E}_{j, x}^{+} / \gamma_{j}-$ $-\eta / \hat{E}_{j, y}^{+} / \gamma_{j}$, в результате получим

$$
\begin{aligned}
& \hat{\mathbf{B}}_{j}^{+}=\mathbf{e}_{x}\left(-\frac{\xi \eta}{\omega \gamma_{j}} \hat{E}_{j, x}^{+}-\left(\frac{\gamma_{j}^{2}+\eta^{2}}{\omega \gamma_{j}}\right) \hat{E}_{j, y}^{+}\right) \\
& +\mathbf{e}_{y}\left(\left(\frac{\gamma_{j}^{2}+\xi^{2}}{\omega \gamma_{j}}\right) \hat{E}_{j, x}^{+}+\frac{\xi \eta}{\omega \gamma_{j}} \hat{E}_{j, y}^{+}\right)+\mathbf{e}_{z}\left(\frac{\xi}{\omega} \hat{E}_{j, y}^{+}-\frac{\eta}{\omega} \hat{E}_{j, x}^{+}\right),
\end{aligned}
$$

или в матричном виде

$$
\left(\begin{array}{c}
\hat{B}_{j, x}^{+} \\
\hat{B}_{j, y}^{+} \\
\hat{B}_{j, z}^{+}
\end{array}\right)=\left(\begin{array}{cc}
-\xi \eta / \omega \gamma_{j} & -\left(\gamma_{j}^{2}+\eta^{2}\right) / \omega \gamma_{j} \\
\left(\gamma_{j}^{2}+\xi^{2}\right) / \omega \gamma_{j} & \xi \eta / \omega \gamma_{j} \\
-\eta / \omega & \xi / \omega
\end{array}\right)\left(\begin{array}{c}
\hat{E}_{j, x}^{+} \\
\hat{E}_{j, y}^{+}
\end{array}\right) .
$$

Аналогично для $\mathbf{B}_{j}^{-}$найдем

$$
\left(\begin{array}{c}
\hat{B}_{j, x}^{-} \\
\hat{B}_{j, y}^{-} \\
\hat{B}_{j, z}^{-}
\end{array}\right)=\left(\begin{array}{cc}
\xi \eta / \omega \gamma_{j} & \left(\gamma_{j}^{2}+\eta^{2}\right) / \omega \gamma_{j} \\
-\left(\gamma_{j}^{2}+\xi^{2}\right) / \omega \gamma_{j} & -\xi \eta / \omega \gamma_{j} \\
-\eta / \omega & \xi / \omega
\end{array}\right)\left(\begin{array}{c}
\hat{E}_{j, x}^{-} \\
\hat{E}_{j, y}^{-}
\end{array}\right) .
$$

Тогда общее решение для магнитного поля в рассматриваемой области с номером $j$ (пленке с номером $j-1$ ) можно записать в виде

$$
\begin{aligned}
& \mathbf{B}_{j}(x, y, z)= \\
& =\frac{1}{(2 \pi)^{2}} \int_{-\infty}^{+\infty} \int_{-\infty}^{+\infty}\left(\begin{array}{cc}
-\xi \eta / \omega \gamma_{j} & -\left(\gamma_{j}^{2}+\eta^{2}\right) / \omega \gamma_{j} \\
\left(\gamma_{j}^{2}+\xi^{2}\right) / \omega \gamma_{j} & \xi \eta / \omega \gamma_{j} \\
-\eta / \omega & \xi / \omega
\end{array}\right) \\
& \times\left(\begin{array}{c}
\hat{E}_{j, x}^{+} \\
\hat{E}_{j, y}^{+}
\end{array}\right) e^{i \gamma_{j}\left(z-z_{j-1}\right)} e^{i(\xi x+\eta y)} d \xi d \eta \\
& +\frac{1}{(2 \pi)^{2}} \int_{-\infty}^{+\infty}\left(\begin{array}{cc}
\xi \eta / \omega \gamma_{j} & \left(\gamma_{j}^{2}+\eta^{2}\right) / \omega \gamma_{j} \\
-\left(\gamma_{j}^{2}+\xi^{2}\right) / \omega \gamma_{j} & -\xi \eta / \omega \gamma_{j} \\
-\eta / \omega & \xi / \omega
\end{array}\right) \\
& \times\left(\begin{array}{c}
\hat{E}_{j, x}^{-} \\
\hat{E}_{j, y}^{-}
\end{array}\right) e^{-i \gamma_{j}\left(z-z_{j}\right)} e^{i(\xi x+\eta y)} d \xi d \eta .
\end{aligned}
$$

Из (9) и (10) следует, что тангенциальные составляющие фурье-образов полей на границах области $j$ можно представить в виде

$$
\begin{aligned}
& \left.\left(\begin{array}{c}
\tilde{E}_{j, x} \\
\tilde{E}_{j, y} \\
\tilde{B}_{j, x} / \mu_{j} \\
\tilde{B}_{j, y} / \mu_{j}
\end{array}\right)\right|_{z=z_{j-1}}= \\
& =\left(\begin{array}{cccc}
1 & 0 & e^{i \gamma_{j} d_{j-1}} & 0 \\
0 & 1 & 0 & e^{i \gamma_{j} d_{j-1}} \\
-\frac{\xi \eta}{\omega \mu_{j} \gamma_{j}} & -\frac{\left(\gamma_{j}^{2}+\eta^{2}\right)}{\omega \mu_{j} \gamma_{j}} & \frac{\xi \eta}{\omega \mu_{j} \gamma_{j}} e^{i \gamma_{j} d_{j-1}} & \frac{\left(\gamma_{j}^{2}+\eta^{2}\right)}{\omega \mu_{j} \gamma_{j}} e^{i \gamma_{j} d_{j-1}} \\
\frac{\left(\gamma_{j}^{2}+\xi^{2}\right)}{\omega \mu_{j} \gamma_{j}} & \frac{\xi \eta}{\omega \mu_{j} \gamma_{j}} & -\frac{\left(\gamma_{j}^{2}+\xi^{2}\right)}{\omega \mu_{j} \gamma_{j}} e^{i \gamma_{j} d_{j-1}} & -\frac{\xi \eta}{\omega \mu_{j} \gamma_{j}} e^{i \gamma_{j} d_{j-1}}
\end{array}\right)
\end{aligned}
$$$$
\times\left(\begin{array}{c}
\hat{E}_{j, x}^{+} \\
\hat{E}_{j, y}^{+} \\
\hat{E}_{j, x}^{-} \\
\hat{E}_{j, y}^{-}
\end{array}\right)
$$$$
\left.\left(\begin{array}{c}
\tilde{E}_{j, x} \\
\tilde{E}_{j, y} \\
\tilde{B}_{j, x} / \mu_{j} \\
\tilde{B}_{j, y} / \mu_{j}
\end{array}\right)\right|_{z=z_{j}}=
$$$$
=\left(\begin{array}{cccc}
e^{i \gamma_{j} d_{j-1}} & 0 & 1 & 0 \\
0 & e^{i \gamma_{j} d_{j-1}} & 0 & 1 \\
-\frac{\xi \eta}{\omega \mu_{j} \gamma_{j}} e^{i \gamma_{j} d_{j-1}} & -\frac{\left(\gamma_{j}^{2}+\eta^{2}\right)}{\omega \mu_{j} \gamma_{j}} e^{i \gamma_{j} d_{j-1}} & \frac{\xi \eta}{\omega \mu_{j} \gamma_{j}} & \frac{\left(\gamma_{j}^{2}+\eta^{2}\right)}{\omega \mu_{j} \gamma_{j}} \\
\frac{\left(\gamma_{j}^{2}+\xi^{2}\right)}{\omega \mu_{j} \gamma_{j}} e^{i \gamma_{j} d_{j-1}} & \frac{\xi \eta}{\omega \mu_{j} \gamma_{j}} e^{i \gamma_{j} d_{j-1}} & -\frac{\left(\gamma_{j}^{2}+\xi^{2}\right)}{\omega \mu_{j} \gamma_{j}} & -\frac{\xi \eta}{\omega \mu_{j} \gamma_{j}}
\end{array}\right)
$$

$$
\times\left(\begin{array}{c}
\hat{E}_{j, x}^{+} \\
\hat{E}_{j, y}^{+} \\
\hat{E}_{j, x}^{-} \\
\hat{E}_{j, y}^{-}
\end{array}\right)
$$

где $d_{j-1}=z_{j}-z_{j-1}$.

Вводя вектор-столбец $\hat{\mathfrak{E}}_{j}=\left(\hat{E}_{j, x}^{+} ; \hat{E}_{j, y}^{+} ; \hat{E}_{j, x}^{-} ; \hat{E}_{j, y}^{-}\right)^{T}$, запишем полученные выражения в матричном виде:

$$
\left.\left(\begin{array}{c}
\tilde{E}_{j, x} \\
\tilde{E}_{j, y} \\
\tilde{B}_{j, x} / \mu_{j} \\
\tilde{B}_{j, y} / \mu_{j}
\end{array}\right)\right|_{z=z_{j-1}}=\left(\begin{array}{cc}
\mathbf{I} & e^{i \gamma_{j} d_{j-1}} \mathbf{I} \\
\mathbf{G}_{j} & -e^{i \gamma_{j} d_{j-1}} \mathbf{G}_{j}
\end{array}\right) \hat{\mathfrak{E}}_{j},
$$




$$
\left.\left(\begin{array}{c}
\tilde{E}_{j, x} \\
\tilde{E}_{j, y} \\
\tilde{B}_{j, x} / \mu_{j} \\
\tilde{B}_{j, y} / \mu_{j}
\end{array}\right)\right|_{z=z_{j}}=\left(\begin{array}{cc}
e^{i \gamma_{j} d_{j-1} \mathbf{I}} & \mathbf{I} \\
e^{i \gamma_{j} d_{j-1}} \mathbf{G}_{j} & -\mathbf{G}_{j}
\end{array}\right) \hat{\mathfrak{E}}_{j},
$$

где $\mathbf{I}$ - единичная $2 \times 2$ матрица, а матрица представляется в виде

$$
\mathbf{G}_{j}=\left(\begin{array}{cc}
-\frac{\xi \eta}{\omega \mu_{j} \gamma_{j}} & -\frac{\gamma_{j}^{2}+\eta^{2}}{\omega \mu_{j} \gamma_{j}} \\
\frac{\gamma_{j}^{2}+\xi^{2}}{\omega \mu_{j} \gamma_{j}} & \frac{\xi \eta}{\omega \mu_{j} \gamma_{j}}
\end{array}\right)
$$

\section{Распространение электромагнитной волны в многослойной структуре, свободной от сторонних токов}

Рассмотрим теперь многослойную структуру, внутри которой нет сторонних токов. Рассмотрим границу $z=z_{j}$ между областями с номерами $j$ и $j+1$. Непрерывность тангенциальных компонент напряженностей электрического и магнитного полей на этой границе можно записать в виде

$$
\begin{gathered}
E_{j, x}\left(x, y, z_{j}\right)-E_{j+1, x}\left(x, y, z_{j}\right)=0, \\
E_{j, y}\left(x, y, z_{j}\right)-E_{j+1, y}\left(x, y, z_{j}\right)=0, \\
B_{j, x}\left(x, y, z_{j}\right) / \mu_{j}-B_{j+1, x}\left(x, y, z_{j}\right) / \mu_{j+1}=0, \\
B_{j, y}\left(x, y, z_{j}\right) / \mu_{j}-B_{j+1, y}\left(x, y, z_{j}\right) / \mu_{j+1}=0,
\end{gathered}
$$

где электрические и магнитные поля в области $j+1$ выражаются формулами (9) и (10), в которых произведена замена индексов $j \rightarrow j+1$. Так как уравнения Максвелла - линейные уравнения, то граничные условия должны выполняться для каждого члена фурьеразложения, т.е. граничные условия выполняются для фурье-образов полей:

$$
\begin{gathered}
\tilde{E}_{j, x}\left(\xi, \eta, z_{j}\right)-\tilde{E}_{j+1, x}\left(\xi, \eta, z_{j}\right)=0, \\
\tilde{E}_{j, y}\left(\xi, \eta, z_{j}\right)-\tilde{E}_{j+1, y}\left(\xi, \eta, z_{j}\right)=0, \\
\tilde{B}_{j, x}\left(\xi, \eta, z_{j}\right) / \mu_{j}-\tilde{B}_{j+1, x}\left(\xi, \eta, z_{j}\right) / \mu_{j+1}=0, \\
\tilde{B}_{j, y}\left(\xi, \eta, z_{j}\right) / \mu_{j}-\tilde{B}_{j+1, y}\left(\xi, \eta, z_{j}\right) / \mu_{j+1}=0 .
\end{gathered}
$$

Записывая граничные условия (14)-(17) с помощью выражений (11) и (12), получим матричное уравнение на границе $z=z_{j}$ :

$$
\left(\begin{array}{cc}
e^{i \gamma_{j} d_{j-1} \mathbf{I}} & \mathbf{I} \\
e^{i \gamma_{j} d_{j-1}} \mathbf{G}_{j} & -\mathbf{G}_{j}
\end{array}\right) \hat{\mathfrak{E}}_{j}=\left(\begin{array}{cc}
\mathbf{I} & e^{i \gamma_{j+1} d_{j} \mathbf{I}} \\
\mathbf{G}_{j+1} & -e^{i \gamma_{j+1} d_{j}} \mathbf{G}_{\mathbf{j}+\mathbf{1}}
\end{array}\right) \hat{\mathfrak{E}}_{j+1},
$$

где $d_{j-1}=z_{j}-z_{j-1}, d_{j}=z_{j+1}-z_{j}$.

Уравнение (18) можно записать для $j=2, \ldots$ $\ldots,(N-1)$, где $(N+1)$ - общее число областей, $N$ число границ, т.е. для всех границ, исключая первую $(j=1)$ и последнюю $(j=N)$ границы. То есть, исключая границу $z_{1}=0$ и $z_{N}=d_{\text {tot }}=\sum_{m=1}^{N-1} d_{m}$, где $d_{\text {tot }}-$ общая толщина слоистой структуры (сумма толщин пленок, составляющих рассматриваемую структуру).

Общее решение для электрического и магнитного полей в области $j=1$, т.е. в интервале $\left(-\infty, z_{1}\right]$, где $z_{1}=0$, запишем в виде

$\mathbf{E}_{1}(x, y, z)=\frac{1}{(2 \pi)^{2}} \int_{-\infty}^{+\infty} \int_{-\infty}^{+\infty}\left(\begin{array}{cc}1 & 0 \\ 0 & 1 \\ -\xi / \gamma_{1} & -\eta / \gamma_{1}\end{array}\right)$

$\times\left(\begin{array}{c}\hat{E}_{1, x}^{+} \\ \hat{E}_{1, y}^{+}\end{array}\right) e^{i \gamma_{1} z} e^{i(\xi x+\eta y)} d \xi d \eta$

$+\frac{1}{(2 \pi)^{2}} \int_{-\infty-\infty}^{+\infty} \int_{-\infty}^{+\infty}\left(\begin{array}{cc}1 & 0 \\ 0 & 1 \\ \xi / \gamma_{1} & \eta / \gamma_{1}\end{array}\right)\left(\begin{array}{c}\hat{E}_{1, x}^{-} \\ \hat{E}_{1, y}^{-}\end{array}\right) e^{-i \gamma_{1} z} e^{i(\xi x+\eta y)} d \xi d \eta$

$\mathbf{B}_{1}(x, y, z)=$

$=\frac{1}{(2 \pi)^{2}} \int_{-\infty}^{+\infty} \int_{-\infty}^{+\infty}\left(\begin{array}{cc}-\xi \eta / \omega \gamma_{1} & -\left(\gamma_{1}^{2}+\eta^{2}\right) / \omega \gamma_{1} \\ \left(\gamma_{1}^{2}+\xi^{2}\right) / \omega \gamma_{1} & \xi \eta / \omega \gamma_{1} \\ -\eta / \omega & \xi / \omega\end{array}\right)$

$\times\left(\begin{array}{c}\hat{E}_{1, x}^{+} \\ \hat{E}_{1, y}^{+}\end{array}\right) e^{i \gamma_{1} z} e^{i(\xi x+\eta y)} d \xi d \eta$

$+\frac{1}{(2 \pi)^{2}} \int_{-\infty}^{+\infty} \int_{-\infty}^{+\infty}\left(\begin{array}{cc}\xi \eta / \omega \gamma_{1} & \left(\gamma_{1}^{2}+\eta^{2}\right) / \omega \gamma_{1} \\ -\left(\gamma_{1}^{2}+\xi^{2}\right) / \omega \gamma_{1} & -\xi \eta / \omega \gamma_{1} \\ -\eta / \omega & \xi / \omega\end{array}\right)$

$\times\left(\begin{array}{c}\hat{E}_{1, x}^{-} \\ \hat{E}_{1, y}^{-}\end{array}\right) e^{-i \gamma_{1} z} e^{i(\xi x+\eta y)} d \xi d \eta$

Записывая граничные условия на границе $z_{1}=0$, полу- 
чим

плоскослоистой структуры):

$$
\left(\begin{array}{cc}
\mathbf{I} & \mathbf{I} \\
\mathbf{G}_{1} & -\mathbf{G}_{1}
\end{array}\right) \hat{\mathfrak{E}}_{1}=\left(\begin{array}{cc}
\mathbf{I} & e^{i \gamma_{2} d_{1} \mathbf{I}} \\
\mathbf{G}_{2} & -e^{i \gamma_{2} d_{1}} \mathbf{G}_{2}
\end{array}\right) \hat{\mathfrak{E}}_{2} .
$$

Общее решение для электрического и магнитного полей в области $j=N+1$, т.е. в интервале $\left[z_{N},+\infty\right)$, запишем в виде

$$
\begin{aligned}
& \mathbf{E}_{N+1}(x, y, z)=\frac{1}{(2 \pi)^{2}} \int_{-\infty}^{+\infty} \int_{-\infty}^{+\infty}\left(\begin{array}{cc}
1 & 0 \\
0 & 1 \\
-\xi / \gamma_{N+1} & -\eta / \gamma_{N+1}
\end{array}\right) \\
& \times\left(\begin{array}{c}
\hat{E}_{N+1, x}^{+} \\
\hat{E}_{N+1, y}^{+}
\end{array}\right) e^{i \gamma_{N+1}\left(z-z_{N}\right)} e^{i(\xi x+\eta y)} d \xi d \eta \\
& +\frac{1}{(2 \pi)^{2}} \int_{-\infty}^{+\infty} \int_{-\infty}^{+\infty}\left(\begin{array}{cc}
1 & 0 \\
0 & 1 \\
\xi / \gamma_{N+1} & \eta / \gamma_{N+1}
\end{array}\right) \\
& \times\left(\begin{array}{c}
\hat{E}_{N+1, x}^{-} \\
\hat{E}_{N+1, y}^{-}
\end{array}\right) e^{-i \gamma_{N+1}\left(z-z_{N}\right)} e^{i(\xi x+\eta y)} d \xi d \eta,
\end{aligned}
$$

$$
\begin{aligned}
& \mathbf{B}_{N+1}(x, y, z)= \\
& =\frac{1}{(2 \pi)^{2}} \int_{-\infty-\infty}^{+\infty+\infty}\left(\begin{array}{cc}
-\xi \eta / \omega \gamma_{N+1} & -\left(\gamma_{N+1}^{2}+\eta^{2}\right) / \omega \gamma_{N+1} \\
\left(\gamma_{N+1}^{2}+\xi^{2}\right) \omega \gamma_{N+1} & \xi \eta / \omega \gamma_{N+1} \\
-\eta / \omega & \xi / \omega
\end{array}\right) \\
& \times\left(\begin{array}{cc}
\hat{E}_{N+1, x}^{+} \\
\hat{E}_{N+1, y}^{+}
\end{array}\right) e^{i \gamma_{N+1}\left(z-z_{N}\right)} e^{i(\xi x+\eta y)} d \xi d \eta \\
& +\frac{1}{(2 \pi)^{2}} \int_{-\infty-\infty}^{+\infty+\infty}\left(\begin{array}{cc}
\xi \eta / \omega \gamma_{N+1} & \left(\gamma_{N+1}^{2}+\eta^{2}\right) / \omega \gamma_{N+1} \\
-\left(\gamma_{N+1}^{2}+\xi^{2}\right) / \omega \gamma_{N+1} & -\xi \eta / \omega \gamma_{N+1} \\
-\eta / \omega & \xi / \omega
\end{array}\right) \\
& \times\left(\begin{array}{c}
\hat{E}_{N+1, x}^{-} \\
\hat{E}_{N+1, y}^{-}
\end{array}\right) e^{-i \gamma_{N+1}\left(z-z_{N}\right)} e^{i(\xi x+\eta y)} d \xi d \eta .
\end{aligned}
$$

Тогда получим граничные условия на границе $z_{N}$ в виде

$$
\left(\begin{array}{cc}
e^{i \gamma_{N} d_{N-1} \mathbf{I}} & \mathbf{I} \\
e^{i \gamma_{N} d_{N-1}} \mathbf{G}_{N} & -\mathbf{G}_{N}
\end{array}\right) \hat{\mathfrak{E}}_{N}=\left(\begin{array}{cc}
\mathbf{I} & \mathbf{I} \\
\mathbf{G}_{N+1} & -\mathbf{G}_{N+1}
\end{array}\right) \hat{\mathfrak{E}}_{N+1} .
$$

Граничные условия (18), (21) и (24) позволяют связать векторы-столбцы электрического поля в первой и последней областях задачи (т.е. в полупространствах, вне

$$
\begin{aligned}
\mathfrak{E}_{1} & =\left(\begin{array}{cc}
\mathbf{I} & \mathbf{I} \\
\mathbf{G}_{1} & -\mathbf{G}_{1}
\end{array}\right)^{-1} \\
& \times\left(\begin{array}{cc}
\mathbf{I} & e^{i \gamma_{2} d_{1} \mathbf{I}} \\
\mathbf{G}_{2} & -e^{i \gamma_{2} d_{1}} \mathbf{G}_{2}
\end{array}\right) \times\left(\begin{array}{cc}
e^{i \gamma_{2} d_{1} \mathbf{I}} & \mathbf{I} \\
e^{i \gamma_{s} d_{1}} \mathbf{G}_{2} & -\mathbf{G}_{2}
\end{array}\right)^{-1} \times \\
& \times\left(\begin{array}{cc}
\mathbf{I} & e^{i \gamma_{N} d_{N-1} \mathbf{I}} \\
\mathbf{G}_{N} & -e^{-\gamma_{N} d_{N-1}} \mathbf{G}_{N}
\end{array}\right) \times\left(\begin{array}{cc}
e^{i \gamma_{N} d_{N-1} \mathbf{I}} & \mathbf{I} \\
e^{i \gamma_{N} d_{N-1}} \mathbf{G}_{N} & -\mathbf{G}_{N}
\end{array}\right)^{-1} \\
& \times\left(\begin{array}{cc}
\mathbf{I} & \mathbf{I} \\
\mathbf{G}_{N+1} & -\mathbf{G}_{N+1}
\end{array}\right) \hat{\mathfrak{E}}_{N+1}
\end{aligned}
$$

или

$$
\hat{\mathfrak{E}}_{1}=\mathbf{M} \times \hat{\mathfrak{E}}_{N+1},
$$

где матрица $\mathbf{M}$ имеет вид $\mathbf{M}=\mathbf{T}_{1} \times\left(\prod_{m=2}^{N} \mathbf{T}_{m}\right) \times \mathbf{T}_{N+1}$, где

$$
\begin{gathered}
\mathbf{T}_{1}=\left(\begin{array}{cc}
\mathbf{I} & \mathbf{I} \\
\mathbf{G}_{1} & -\mathbf{G}_{1}
\end{array}\right)^{-1}, \\
\mathbf{T}_{m}=\left(\begin{array}{cc}
\mathbf{I} & e^{i \gamma_{m} d_{m-1} \mathbf{I}} \\
\mathbf{G}_{m} & -e^{-i \gamma_{m} d_{m-1}} \mathbf{G}_{m}
\end{array}\right)\left(\begin{array}{cc}
e^{i \gamma_{m} d_{m-1} \mathbf{I}} & \mathbf{I} \\
e^{i \gamma_{m} d_{m-1}} \mathbf{G}_{m} & -\mathbf{G}_{m}
\end{array}\right)^{-1}, \\
\mathbf{T}_{N+1}=\left(\begin{array}{cc}
\mathbf{I} & \mathbf{I} \\
\mathbf{G}_{N+1} & -\mathbf{G}_{N+1}
\end{array}\right) .
\end{gathered}
$$

Если нам известна, например, падающая на плоскослоистую структуру волна, а значит компоненты $\hat{E}_{1, x}^{+}$и $\hat{E}_{1, y}^{+}$вектора-столбца $\hat{\mathfrak{E}}_{1}$, то из уравнения $(25)$ можно найти компоненты $\hat{E}_{1, x}^{-}$и $\hat{E}_{1, y}^{-}$и саму отраженную волну по формулам (19), (20) и компоненты $\hat{E}_{N+1, x}^{+}$и $\hat{E}_{N+1, y}^{+}$вектора-столбца $\hat{\mathfrak{E}}_{N+1}$ и прошедшую волну по формулам (22), (23). Подробности решения таких задач для пространственно ограниченных падающих пучков можно найти, например, в работах [15-17].

\section{Распространение электромагнитной волны в многослойной структуре от элементарного источника стороннего тока}

Пусть имеется точечный излучатель, расположенный в точке $\left(0,0, z_{d}\right)$ в области с номером $s$ (рис. 4). Пусть этот излучатель определяется плотностью стороннего тока (в комплексном представлении $e^{-i \omega t}$ ):

$$
\mathbf{J}(x, y, z)=\left(n_{x} \mathbf{e}_{x}+n_{y} \mathbf{e}_{y}+n_{z} \mathbf{e}_{z}\right) \delta(x) \delta(y) \delta\left(z-z_{d}\right),
$$

где $n_{x}, n_{y}, n_{z}$ - направляющие косинусы вектора тока вдоль осей координат, причем $n_{x}^{2}+n_{y}^{2}+n_{z}^{2}=1$. Тогда 


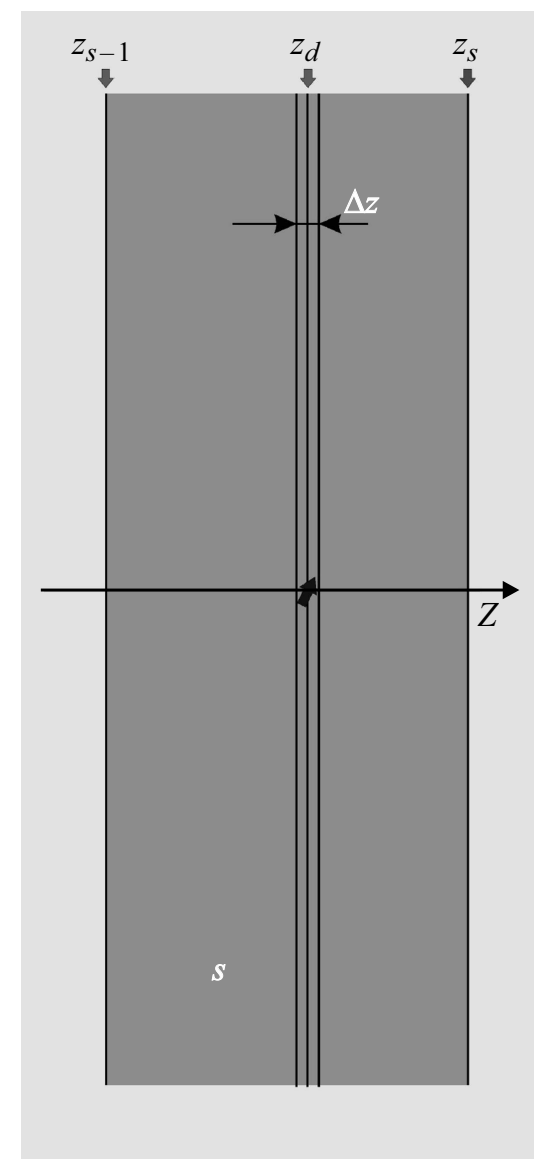

Рис. 4. Точечный излучатель, расположенный в точке $\left(0,0, z_{d}\right)$ в области с номером $s$.

фурье-образы составляющих этого тока определятся следующими выражениями:

$$
\begin{gathered}
\tilde{J}_{s, x}(\xi, \eta, z)=n_{x} \int_{-\infty}^{+\infty} \int_{-\infty}^{+\infty} \delta(x) \delta(y) \delta\left(z-z_{d}\right) e^{-i(\xi x+\eta y)} d x d y \\
=n_{x} \delta\left(z-z_{d}\right) \\
\tilde{J}_{s, y}(\xi, \eta, z)=n_{y} \delta\left(z-z_{d}\right) \\
\tilde{J}_{s, z}(\xi, \eta, z)=n_{z} \delta\left(z-z_{d}\right)
\end{gathered}
$$

Пусть этот элементарный источник тока находится в бесконечно тонком слое $\left(z_{d}-\Delta z / 2, z_{d}+\Delta z / 2\right)$. Тогда уравнения Максвелла (1) и (2) для фурье-образов полей можно записать (при $\Delta z \rightarrow 0$ ) в виде

$$
\left\{\begin{array}{l}
i \eta \tilde{E}_{s, z}-\frac{\Delta E_{s, y}}{\Delta z}=i \omega \tilde{B}_{s, x} \\
\frac{\Delta \tilde{E}_{s, x}}{\Delta z}-i \xi \tilde{E}_{s, z}=i \omega \tilde{B}_{s, y} \\
i \xi \tilde{E}_{s, y}-i \eta \tilde{E}_{s, x}=i \omega \tilde{B}_{s, z}
\end{array}\right.
$$

$$
\left\{\begin{array}{l}
i \eta \tilde{B}_{s, z}-\frac{\Delta \tilde{B}_{s, y}}{\Delta z}=\mu_{s}\left(-i \omega \varepsilon_{s} \tilde{E}_{s, x}+n_{x} \delta\left(z-z_{d}\right)\right), \\
\frac{\Delta \tilde{B}_{s, x}}{\Delta z}-i \xi \tilde{B}_{s, z}=\mu_{s}\left(-i \omega \varepsilon_{s} \tilde{E}_{s, y}+n_{y} \delta\left(z-z_{d}\right)\right), \\
i \xi \tilde{B}_{s, y}-i \eta \tilde{B}_{s, x}=\mu_{s}\left(-i \omega \varepsilon_{s} \tilde{E}_{s, z}+n_{z} \delta\left(z-z_{d}\right)\right) .
\end{array}\right.
$$

Так как $z$-компоненты полей из уравнений (26) и (27) можно выразить через $x$ - и $y$-компоненты по формулам

$$
\begin{gathered}
\tilde{B}_{s, z}=\frac{\xi}{\omega} \tilde{E}_{s, y}-\frac{\eta}{\omega} \tilde{E}_{s, x} \text { и } \\
\tilde{E}_{s, z}=-\frac{\xi}{\omega \varepsilon_{s} \mu_{s}} \tilde{B}_{s, y}+\frac{\eta}{\omega \varepsilon_{s} \mu_{s}} \tilde{B}_{s, x}+\frac{1}{i \omega \varepsilon_{s}} \tilde{J}_{s, z},
\end{gathered}
$$

то для $x$ - и $y$-компонент напряженностей электрического и магнитного полей получим

$$
\left\{\begin{aligned}
\Delta \tilde{E}_{s, x}= & \left(\frac{i \xi \eta}{\omega \varepsilon_{s} \mu_{s}} \tilde{B}_{s, x}+i\left(\omega-\frac{\xi^{2}}{\omega \varepsilon_{s} \mu_{s}}\right) \tilde{B}_{s, y}\right. \\
& \left.+\frac{\xi}{\omega \varepsilon_{s}} n_{z} \delta\left(z-z_{d}\right)\right) \delta z, \\
& \left.\quad+\frac{\eta}{\omega \varepsilon_{s}} n_{z} \delta\left(z-z_{d}\right)\right) \Delta z, \\
& \left(i\left(\frac{\eta^{2}}{\omega \varepsilon_{s} \mu_{s}}-\omega\right) \tilde{B}_{s, x}-\frac{i \xi \eta}{\omega \varepsilon_{s} \mu_{s}} \tilde{B}_{s, y}\right. \\
\frac{\Delta \tilde{B}_{s, x}}{\mu_{s}}= & \left(-\frac{i \xi \eta}{\omega \mu_{s}} \tilde{E}_{s, z}+i\left(\frac{\xi^{2}}{\omega \mu_{s}}-\omega \varepsilon_{s}\right) \tilde{E}_{s, y}+n_{y} \delta\left(z-z_{d}\right)\right) \Delta z, \\
\frac{\Delta \tilde{B}_{s, y}}{\mu_{s}}= & \left(i\left(\omega \varepsilon_{s}-\frac{\eta^{2}}{\omega \mu_{s}}\right) \tilde{E}_{s, x}+\frac{i \xi \eta}{\omega \mu_{s}} \tilde{E}_{s, y}-n_{x} \delta\left(z-z_{d}\right)\right) \Delta z .
\end{aligned}\right.
$$

Тогда в пределе $\Delta z \rightarrow 0$ скачок тангенциальных компонент напряженностей электрического и магнитного полей при переходе через бесконечно тонкий слой с током равен

$$
\left\{\begin{array}{l}
\Delta \tilde{E}_{s, x} \underset{\Delta z \rightarrow 0}{\longrightarrow} n_{z} \xi / \omega \varepsilon_{s} \\
\Delta \tilde{E}_{s, y} \underset{\Delta z \rightarrow 0}{\longrightarrow} n_{z} \eta / \omega \varepsilon_{s} \\
\Delta \tilde{B}_{s, x} / \mu_{s} \underset{\Delta z \rightarrow 0}{\longrightarrow} n_{y} \\
\Delta \tilde{B}_{s, y} / \mu_{s} \underset{\Delta z \rightarrow 0}{\longrightarrow}-n_{x}
\end{array}\right.
$$

В матричном виде уравнения, связывающие тангенциальные компоненты полей с двух сторон слоя с током, можно записать в следующем виде:

$$
\left.\left(\begin{array}{c}
\tilde{E}_{s, x} \\
\tilde{E}_{s, y} \\
\tilde{B}_{s, x} / \mu_{s} \\
\tilde{B}_{s, y} / \mu_{s}
\end{array}\right)\right|_{z=z_{d}+0}-\left.\left(\begin{array}{c}
\tilde{E}_{s, x} \\
\tilde{E}_{s, y} \\
\tilde{B}_{s, x} / \mu_{s} \\
\tilde{B}_{s, y} / \mu_{s}
\end{array}\right)\right|_{z=z_{d}-0}=\left(\begin{array}{c}
n_{z} \xi / \omega \varepsilon_{s} \\
n_{z} \eta / \omega \varepsilon_{s} \\
n_{y} \\
-n_{x}
\end{array}\right) .
$$

Особо отметим, что скачок электрических полей при переходе через рассматриваемый бесконечно тонкий слой (при $\Delta z \rightarrow 0)$, вообще говоря, зависит от диэлектрической проницаемости $\varepsilon_{s}$ среды, в которой расположен рассматриваемый источник тока. Но излучение 
источников, у которых есть только составляющие тока вдоль границы, не зависит в явном виде от диэлектрических свойств среды, в которой они расположены.

Выразим теперь левую часть граничного условия (28) через векторы-столбцы $\hat{\mathfrak{E}}_{1}$ и $\hat{\mathfrak{E}}_{N+1}$ полупространств снаружи плоскослоистой структуры. Для этого разобьем область с номером $s$ на две области и обозначим их индексами $l$ и $r$ (левая и правая, если смотреть на рис. 4). Введем векторы-столбцы $\hat{\mathfrak{E}}_{l}$ и $\hat{\mathfrak{E}}_{r}$ в этих областях. Тогда тангенциальные компоненты напряженностей полей в (28) можно выразить как

$$
\begin{aligned}
& \left.\left(\begin{array}{c}
\tilde{E}_{s, x} \\
\tilde{E}_{s, y} \\
\tilde{B}_{s, x} / \mu_{s} \\
\tilde{B}_{s, y} / \mu_{s}
\end{array}\right)\right|_{z=z_{d}-0}=\left(\begin{array}{cc}
e^{i \gamma_{s}\left(z_{d}-z_{s-1}\right)} \mathbf{I} & \mathbf{I} \\
e^{i \gamma_{s}\left(z_{d}-z_{s-1}\right)} \mathbf{G}_{s} & -\mathbf{G}_{s}
\end{array}\right) \hat{\mathfrak{E}}_{l}, \\
& \left.\left(\begin{array}{c}
\tilde{E}_{s, x} \\
\tilde{E}_{s, y} \\
\tilde{B}_{s, x} / \mu_{s} \\
\tilde{B}_{s, y} / \mu_{s}
\end{array}\right)\right|_{z=z_{d}+0}=\left(\begin{array}{cc}
\mathbf{I} & e^{i \gamma_{s}\left(z_{s}-z_{d}\right)} \mathbf{I} \\
\mathbf{G}_{s} & -e^{i \gamma_{s}\left(z_{s}-z_{d}\right)} \mathbf{G}_{s}
\end{array}\right) \hat{\mathfrak{E}}_{r},
\end{aligned}
$$

Кроме того, из (25) следует, что

$$
\hat{\mathfrak{E}}_{1}=\mathbf{Q}_{L} \hat{\mathfrak{E}}_{l},
$$

где

$$
\begin{aligned}
& \mathbf{Q}_{L}=\left(\begin{array}{cc}
\mathbf{I} & \mathbf{I} \\
\mathbf{G}_{1} & -\mathbf{G}_{1}
\end{array}\right)^{-1} \times \\
& \times\left(\begin{array}{cc}
\mathbf{I} & e^{i \gamma_{2} d_{1} \mathbf{I}} \\
\mathbf{G}_{2} & -e^{i \gamma_{2} d_{1}} \mathbf{G}_{2}
\end{array}\right) \times\left(\begin{array}{cc}
e^{i \gamma_{2} d_{1} \mathbf{I}} & \mathbf{I} \\
e^{i \gamma_{2} d_{1}} \mathbf{G}_{2} & -\mathbf{G}_{2}
\end{array}\right)^{-1} \times \\
& \ldots \times\left(\begin{array}{cc}
\mathbf{I} & e^{i \gamma_{s-1} d_{s-2} \mathbf{I}} \\
\mathbf{G}_{s-1} & -e^{i \gamma_{s-1} d_{s-2}} \mathbf{G}_{s-1}
\end{array}\right) \times \\
& \times\left(\begin{array}{cc}
e^{i \gamma_{s-1} d_{s-2} \mathbf{I}} & \mathbf{I} \\
e^{i \gamma_{s-1} d_{s-2} \mathbf{G}_{s-1}} & -\mathbf{G}_{s-1}
\end{array}\right)^{-1} \times\left(\begin{array}{cc}
\mathbf{I} & e^{i \gamma_{s}\left(z_{d}-z_{s-1}\right)} \mathbf{I} \\
\mathbf{G}_{s} & -e^{i \gamma_{s}\left(z_{d}-z_{s-1}\right)} \mathbf{G}_{s}
\end{array}\right),
\end{aligned}
$$

где

$\mathbf{Q}_{R}=\left(\begin{array}{cc}e^{i \gamma_{s}\left(z_{s}-z_{d}\right)} \mathbf{I} & \mathbf{I} \\ e^{i \gamma_{s}\left(z_{s}-z_{d}\right)} \mathbf{G}_{s} & -\mathbf{G}_{s}\end{array}\right)^{-1} \times$

$\times\left(\begin{array}{cc}\mathbf{I} & e^{i \gamma_{s+1} d_{s}} \mathbf{I} \\ \mathbf{G}_{s+1} & -e^{i \gamma_{s+1} d_{s}} \mathbf{G}_{s+1}\end{array}\right) \times\left(\begin{array}{cc}e^{i \gamma_{s+1} d_{s}} \mathbf{I} & \mathbf{I} \\ e^{i \gamma_{s+1} d_{s}} \mathbf{G}_{s+1} & -\mathbf{G}_{s+1}\end{array}\right)^{-1} \times$

$\ldots \times\left(\begin{array}{cc}\mathbf{I} & e^{i \gamma_{N} d_{N-1} \mathbf{I}} \\ \mathbf{G}_{N} & -e^{i \gamma_{N} d_{N-1}} \mathbf{G}_{N}\end{array}\right) \times$

$\times\left(\begin{array}{cc}e^{i \gamma_{N} d_{N-1} \mathbf{I}} & \mathbf{I} \\ e^{i \gamma_{N} d_{N-1}} \mathbf{G}_{N} & -\mathbf{G}_{N}\end{array}\right)^{-1} \times\left(\begin{array}{cc}\mathbf{I} & \mathbf{I} \\ \mathbf{G}_{N+1} & -\mathbf{G}_{N+1}\end{array}\right)$.
Подставляя (31), (32) в (29), (30) и затем полученные выражения в (28), получаем

$$
\mathbf{H}_{R} \times \hat{\mathfrak{E}}_{N+1}=\mathbf{H}_{L} \times \hat{\mathfrak{E}}_{1}+\mathbf{V},
$$

где $\mathbf{V}=\left(n_{z} \xi / \omega \varepsilon_{s} ; n_{z} \eta / \omega \varepsilon_{s} ; n_{y} ;-n_{x}\right)^{T}-$ вектор-столбец, характеризующий возбуждающее воздействие на систему стороннего элементарного тока, а матрицы $\mathbf{H}_{R}$ и $\mathbf{H}_{L}$ характеризуют отклик на внешнее возбуждение слоистой структуры справа и слева излучателя и выражаются следующим образом:

$$
\begin{gathered}
\mathbf{H}_{R}=\left(\begin{array}{cc}
\mathbf{I} & e^{i \gamma_{s}\left(z_{s}-z_{d}\right)} \mathbf{I} \\
\mathbf{G}_{s} & -e^{i \gamma_{s}\left(z_{s}-z_{d}\right)} \mathbf{G}_{s}
\end{array}\right) \mathbf{Q}_{R}=\mathbf{T}_{R} \times\left(\prod_{m=s+1}^{N} \mathbf{T}_{m}\right) \times T_{N+1}, \\
\mathbf{H}_{L}=\left(\begin{array}{cc}
e^{i \gamma_{s}\left(z_{d}-z_{s-1}\right)} \mathbf{I} & \mathbf{I} \\
e^{i \gamma_{s}\left(z_{d}-z_{s-1}\right)} \mathbf{G}_{s} & -\mathbf{G}_{s}
\end{array}\right)\left(\mathbf{Q}_{L}\right)^{-1} \\
=\left(\mathbf{T}_{1} \times\left(\prod_{m=2}^{s-1} \mathbf{T}_{m}\right) \times T_{L}\right)^{-1}
\end{gathered}
$$

где матрицы $\mathbf{T}_{m}$ при $m \neq s$ выражаются формулой

$$
\mathbf{T}_{m}=\left(\begin{array}{cc}
\mathbf{I} & e^{i \gamma_{m} d_{m-1} \mathbf{I}} \\
\mathbf{G}_{m} & -e^{i \gamma_{m} d_{m-1}} \mathbf{G}_{m}
\end{array}\right)\left(\begin{array}{cc}
e^{i \gamma_{m} d_{m-1} \mathbf{I}} & \mathbf{I} \\
e^{i \gamma_{m} d_{m-1}} \mathbf{G}_{m} & -\mathbf{G}_{m}
\end{array}\right)^{-1}
$$

а матрицы $\mathbf{T}_{L}$ и $\mathbf{T}_{R}-$ формулами

$$
\begin{gathered}
\mathbf{T}_{L}=\left(\begin{array}{cc}
\mathbf{I} & e^{i \gamma_{s}\left(z_{d}-z_{s-1}\right)} \mathbf{I} \\
\mathbf{G}_{s} & -e^{i \gamma_{s}\left(z_{d}-z_{s-1}\right)} \mathbf{G}_{s}
\end{array}\right)\left(\begin{array}{cc}
e^{i \gamma_{s}\left(z_{d}-z_{s-1}\right)} \mathbf{I} & \mathbf{I} \\
e^{i \gamma_{s}\left(z_{d}-z_{s-1}\right)} \mathbf{G}_{s} & -\mathbf{G}_{s}
\end{array}\right)^{-1}, \\
\mathbf{T}_{m}=\left(\begin{array}{cc}
\mathbf{I} & e^{i \gamma_{s}\left(z_{s}-z_{d}\right)} \mathbf{I} \\
\mathbf{G}_{s} & -e^{i \gamma_{s}\left(z_{s}-z_{d}\right)} \mathbf{G}_{s}
\end{array}\right)\left(\begin{array}{cc}
e^{i \gamma_{s}\left(z_{s}-z_{d}\right)} \mathbf{I} & \mathbf{I} \\
e^{i \gamma_{s}\left(z_{s}-z_{d}\right)} \mathbf{G}_{s} & -\mathbf{G}_{s}
\end{array}\right)^{-1} .
\end{gathered}
$$

В вышеприведенных формулах матрицы $\mathbf{G}_{s}$ выражаются по формулам (13).

Далее в рассматриваемой задаче источники полей находятся исключительно внутри плоскослоистой структуры. Поэтому в столбцах $\hat{\mathfrak{E}}_{1}$ и $\hat{\mathfrak{E}}_{N+1}$ есть только компоненты волн, идущие от плоскослоистой структуры. Поэтому эти столбцы имеют вид

$$
\begin{gathered}
\hat{\mathfrak{E}}_{1}=\left(0 ; 0 ; \hat{E}_{1, x}^{-} ; \hat{E}_{1, y}^{-}\right)^{T}, \\
\hat{\mathfrak{E}}_{N+1}=\left(\hat{E}_{N+1, x}^{+} ; \hat{E}_{N+1, y}^{+} ; 0 ; 0\right)^{T} .
\end{gathered}
$$

Чтобы получить оставшиеся, отличные от нуля компоненты $\hat{\mathfrak{E}}_{1}$ и $\hat{\mathfrak{E}}_{N+1}$, разобьем матрицы $\mathbf{H}_{R}$ и $\mathbf{H}_{L}$ каждую на четыре $2 \times 1$ подматрицы $\mathbf{H}_{R A}, \mathbf{H}_{R B}, \mathbf{H}_{R C}, \mathbf{H}_{R D}$ и $\mathbf{H}_{L A}$, $\mathbf{H}_{L B}, \mathbf{H}_{L C}, \mathbf{H}_{L D}$ следующим образом:

$$
\mathbf{H}_{R}=\left(\begin{array}{ll}
\mathbf{H}_{R A} & \mathbf{H}_{R B} \\
\mathbf{H}_{R C} & \mathbf{H}_{R D}
\end{array}\right) \quad \text { и } \quad \mathbf{H}_{L}=\left(\begin{array}{ll}
\mathbf{H}_{L A} & \mathbf{H}_{L B} \\
\mathbf{H}_{L C} & \mathbf{H}_{L D}
\end{array}\right),
$$


тогда уравнение (33) примет вид

$$
\left(\begin{array}{cc}
\mathbf{H}_{R A} & \mathbf{H}_{R B} \\
\mathbf{H}_{R C} & \mathbf{H}_{R D}
\end{array}\right) \times\left(\begin{array}{c}
\hat{E}_{N+1, x}^{+} \\
\hat{E}_{N+1, y}^{+} \\
0 \\
0
\end{array}\right)=\left(\begin{array}{cc}
\mathbf{H}_{L A} & \mathbf{H}_{L B} \\
\mathbf{H}_{L C} & \mathbf{H}_{L D}
\end{array}\right) \times\left(\begin{array}{c}
0 \\
0 \\
\hat{E}_{1, x}^{-} \\
\hat{E}_{1, y}^{-}
\end{array}\right)+\mathbf{V} .
$$

Если еще разбить вектор $\mathbf{V}=\left(V_{1} ; V_{2} ; V_{3} ; V_{4}\right)^{T}$ на $\mathbf{V}_{A}=\left(V_{1}, V_{2}\right)^{T}$ и $\mathbf{V}_{B}=\left(V_{3}, V_{4}\right)^{T}$, то уравнение (34) можно представить следующей системой из двух матричных уравнений:

$$
\begin{aligned}
& \mathbf{H}_{R A}\left(\begin{array}{c}
\hat{E}_{N+1, x}^{+} \\
\hat{E}_{N+1, y}^{+}
\end{array}\right)=\mathbf{H}_{L B}\left(\begin{array}{c}
\hat{E}_{1, x}^{-} \\
\hat{E}_{1, y}^{-}
\end{array}\right)+\mathbf{V}_{A}, \\
& \mathbf{H}_{R C}\left(\begin{array}{c}
\hat{E}_{N+1, x}^{+} \\
\hat{E}_{N+1, y}^{+}
\end{array}\right)=\mathbf{H}_{L D}\left(\begin{array}{c}
\hat{E}_{1, x}^{-} \\
\hat{E}_{1, y}^{-}
\end{array}\right)+\mathbf{V}_{B} .
\end{aligned}
$$

Полученные уравнения можно снова объединить в одно матричное $4 \times 4$ уравнение:

$$
\left(\begin{array}{cc}
-\mathbf{H}_{L B} & \mathbf{H}_{R A} \\
-\mathbf{H}_{L D} & \mathbf{H}_{R C}
\end{array}\right) \hat{\mathfrak{E}}_{\mathrm{out}}=\mathbf{V}
$$

$\stackrel{\text { где }}{\hat{\mathfrak{E}}_{\text {out }}}=\left(\hat{E}_{1, x}^{-} ; \hat{E}_{1, y}^{-} ; \hat{E}_{N+1, x}^{+} ; \hat{E}_{N+1, y}^{+}\right)^{T}$.

вектор-столбец

Решая это уравнение, найдем $\hat{E}_{1, x}^{-}, \hat{E}_{1, y}^{-}$и $\hat{E}_{N+1, x}^{+}$, $\hat{E}_{N+1, y}^{+}$, а значит, уходящую из плоскослоистой структуры влево волну (в направлении $z \rightarrow-\infty$ ) по формулам

$$
\begin{gathered}
\mathbf{E}_{1}(x, y, z)=\frac{1}{(2 \pi)^{2}} \int_{-\infty}^{+\infty} \int_{-\infty}^{+\infty}\left(\begin{array}{cc}
1 & 0 \\
0 & 1 \\
\xi / \gamma_{1} & \eta / \gamma_{1}
\end{array}\right) \\
\times\left(\begin{array}{c}
\hat{E}_{1, x}^{-} \\
\hat{E}_{1, y}^{-}
\end{array}\right) e^{-i \gamma_{1} z} e^{i(\xi x+\eta y)} d \xi d \eta, \\
\mathbf{B}_{1}(x, y, z)= \\
=\frac{1}{(2 \pi)^{2}} \int_{-\infty}^{+\infty} \int_{-\infty}^{+\infty}\left(\begin{array}{cc}
\eta \eta / \omega \gamma_{1} & \left(\gamma_{1}^{2}+\eta^{2}\right) / \omega \gamma_{1} \\
-\left(\gamma_{1}^{2}+\xi \xi^{2}\right) / \omega \gamma_{1} & -\xi \eta / \omega \gamma_{1} \\
-\eta / \omega & \xi / \omega
\end{array}\right) \\
\times\left(\begin{array}{c}
\hat{E}_{1, x}^{-} \\
\hat{E}_{1, y}^{-}
\end{array}\right) e^{-i \gamma_{1} z} e^{i(\xi x+\eta y)} d \xi d \eta
\end{gathered}
$$

и волну вправо от плоскослоистой структуры (в направлении $z \rightarrow+\infty)$ по формулам

$$
\begin{aligned}
\mathbf{E}_{N+1}(x, y, z)= & \frac{1}{(2 \pi)^{2}} \int_{-\infty}^{+\infty} \int_{-\infty}^{+\infty}\left(\begin{array}{cc}
1 & 0 \\
0 & 1 \\
-\xi / \gamma_{N+1} & -\eta / \gamma_{N+1}
\end{array}\right) \\
& \times\left(\begin{array}{c}
\hat{E}_{N+1, x}^{+} \\
\hat{E}_{N+1, y}^{+}
\end{array}\right) e^{i \gamma_{N+1}\left(z-z_{N}\right)} e^{i(\xi x+\eta y)} d \xi d \eta,
\end{aligned}
$$

$\mathbf{B}_{N+1}(x, y)=$,

$$
\begin{aligned}
& =\frac{1}{(2 \pi)^{2}} \int_{-\infty-\infty}^{+\infty} \int_{-\infty}^{+\infty}\left(\begin{array}{cc}
-\xi \eta / \omega \gamma_{N+1} & -\left(\gamma_{N+1}^{2}+\eta^{2}\right) / \omega \gamma_{N+1} \\
\left(\gamma_{N+1}^{2}+\xi^{2}\right) / \omega \gamma_{N+1} & \xi \eta / \omega \gamma_{N+1} \\
-\eta / \omega & \xi / \omega
\end{array}\right) \\
& \times\left(\begin{array}{c}
\hat{E}_{N+1, x}^{+} \\
\hat{E}_{N+1, y}^{+}
\end{array}\right) e^{i \gamma_{N+1}\left(z-z_{N}\right)} e^{i(\xi x+\eta y)} d \xi d \eta .
\end{aligned}
$$

Наконец, при необходимости, зная $\hat{\mathfrak{E}}_{1}$ и $\hat{\mathfrak{E}}_{N+1}$, можно найти векторы-столбцы поля в любой внутренней области $\hat{\mathfrak{E}}_{j}$, так как они однозначно определяются граничными условиями. После этого электромагнитные поля в любой из этих областей могут быть найдены по формулам (9), (10). Таким образом, электромагнитные поля будут определены во всем пространстве.

\section{Излучение элементарного диполя, расположенного на границе двух полупространств}

Пусть теперь рассматривается простейшая задача точечный источник на границе двух разных однородных полупространств. Тогда $N=1, z_{0}=0, \mathbf{H}_{R}=\mathbf{T}_{2}$, $\mathbf{H}_{L}=\left(\mathbf{T}_{1}\right)^{-1}$ и уравнение (33) примет вид

$$
\mathbf{T}_{2} \times \hat{\mathfrak{E}}_{2}=\left(\mathbf{T}_{1}\right)^{-1} \times \hat{\mathfrak{E}}_{1}+\mathbf{V},
$$

где $\mathbf{V}=\left(n_{z} \xi / \omega \varepsilon_{s} ; n_{z} \eta / \omega \varepsilon_{s} ; n_{y},-n_{x}\right)^{T}$

$$
\mathbf{T}_{1}=\left(\begin{array}{cc}
\mathbf{I} & \mathbf{I} \\
\mathbf{G}_{1} & -\mathbf{G}_{1}
\end{array}\right)^{-1}, \mathbf{T}_{2}=\left(\begin{array}{cc}
\mathbf{I} & \mathbf{I} \\
\mathbf{G}_{2} & -\mathbf{G}_{2}
\end{array}\right) .
$$

В выражении для $\mathbf{V}$ будем полагать $\varepsilon_{s}=\varepsilon_{1}$, если источник находится в первой среде бесконечно близко к границе, и $\varepsilon_{s}=\varepsilon_{2}$, если во второй среде.

Тогда, получим из (39) уравнение

$$
\left(\begin{array}{cc}
\mathbf{I} & \mathbf{I} \\
\mathbf{G}_{2} & -\mathbf{G}_{2}
\end{array}\right) \times \hat{\mathfrak{E}}_{2}=\left(\begin{array}{cc}
\mathbf{I} & \mathbf{I} \\
\mathbf{G}_{1} & -\mathbf{G}_{1}
\end{array}\right) \times \hat{\mathfrak{E}}_{1}+\mathbf{V} .
$$

Далее в рассматриваемой задаче источник полей находится на границе, поэтому в столбцах $\mathfrak{E}_{1}$ и $\mathfrak{E}_{2}$ есть 
только компоненты волн, идущие от плоскослоистой структуры. Поэтому эти столбцы имеют вид

$$
\hat{\mathfrak{E}}_{1}=\left(0 ; 0 ; \hat{E}_{1, x}^{-} ; \hat{E}_{1, y}^{-}\right)^{T}, \quad \hat{\mathfrak{E}}_{2}=\left(\hat{E}_{2, x}^{+} ; \hat{E}_{2, y}^{+} ; 0 ; 0\right)^{T} .
$$

Чтобы получить оставшиеся компоненты $\hat{\mathfrak{E}}_{1}$ и $\hat{\mathfrak{E}}_{2}$, разобьем матрицы $\mathbf{H}_{R}$ и $\mathbf{H}_{L}$ каждую на четыре $2 \times 2$ подматрицы $\mathbf{H}_{R A}, \mathbf{H}_{R B}, \mathbf{H}_{R C}, \mathbf{H}_{R D}$ и $\mathbf{H}_{L A}, \mathbf{H}_{L B}, \mathbf{H}_{L C}, \mathbf{H}_{L D}$ следующим образом:

$$
\begin{gathered}
\mathbf{H}_{R}\left(\begin{array}{ll}
\mathbf{H}_{R A} & \mathbf{H}_{R B} \\
\mathbf{H}_{R C} & \mathbf{H}_{R D}
\end{array}\right)=\left(\begin{array}{cc}
\mathbf{I} & \mathbf{I} \\
\mathbf{G}_{2} & -\mathbf{G}_{2}
\end{array}\right) \text { и } \\
\mathbf{H}_{L}=\left(\begin{array}{ll}
\mathbf{H}_{L A} & \mathbf{H}_{L B} \\
\mathbf{H}_{L C} & \mathbf{H}_{L D}
\end{array}\right)=\left(\begin{array}{cc}
\mathbf{I} & \mathbf{I} \\
\mathbf{G}_{1} & -\mathbf{G}_{1}
\end{array}\right) .
\end{gathered}
$$

Как и ранее, после введения вектор-столбца $\hat{\mathfrak{E}}_{\text {out }}=\left(\hat{E}_{1, x}^{-} ; \hat{E}_{1, y}^{-} ; \hat{E}_{2, x}^{+} ; \hat{E}_{2, y}^{+}\right)^{T}$ уравнение (35) примет вид

$$
\left(\begin{array}{cc}
-\mathbf{I} & \mathbf{I} \\
\mathbf{G}_{1} & \mathbf{G}_{2}
\end{array}\right) \hat{\mathfrak{E}}_{\mathrm{out}}=\mathbf{V},
$$

или в явном виде

$$
\begin{gathered}
\left(\begin{array}{cccc}
-1 & 0 & 1 & 0 \\
0 & -1 & 0 & 1 \\
-\frac{\xi \eta}{\omega \mu_{1} \gamma_{1}} & -\frac{\gamma_{1}^{2}+\eta^{2}}{\omega \mu_{1} \gamma_{1}} & -\frac{\xi \eta}{\omega \mu_{2} \gamma_{2}} & -\frac{\gamma_{2}^{2}+\eta^{2}}{\omega \mu_{2} \gamma_{2}} \\
\frac{\gamma_{1}^{2}+\xi^{2}}{\omega \mu_{1} \gamma_{1}} & \frac{\xi \eta}{\omega \mu_{1} \gamma_{1}} & \frac{\gamma_{2}^{2}+\xi^{2}}{\omega \mu_{2} \gamma_{2}} & \frac{\xi \eta}{\omega \mu_{2} \gamma_{2}}
\end{array}\right) \\
\times\left(\begin{array}{c}
\hat{E}_{1, x}^{-} \\
\hat{E}_{1, y}^{-} \\
\hat{E}_{2, x}^{+} \\
\hat{E}_{2, y}^{+}
\end{array}\right)=\left(\begin{array}{c}
n_{z} \xi / \omega \varepsilon_{s} \\
n_{z} \eta / \omega \varepsilon_{s} \\
n_{y} \\
-n_{x}
\end{array}\right) .
\end{gathered}
$$

Очень часто рассматриваются немагнитные среды, для которых $\mu_{1}=\mu_{2}=\mu_{0}$, тогда уравнение (41) упрощается и записывается в виде

$$
\begin{gathered}
\left(\begin{array}{cccc}
-1 & 0 & 1 & 0 \\
0 & -1 & 0 & 1 \\
-\frac{\xi \eta}{\omega \mu_{0} \gamma_{1}} & -\frac{\gamma_{1}^{2}+\eta^{2}}{\omega \mu_{0} \gamma_{1}} & -\frac{\xi \eta}{\omega \mu_{0} \gamma_{2}} & -\frac{\gamma_{2}^{2}+\eta^{2}}{\omega \mu_{0} \gamma_{2}} \\
\frac{\gamma_{1}^{2}+\xi^{2}}{\omega \mu_{0} \gamma_{1}} & \frac{\xi \eta}{\omega \mu_{0} \gamma_{1}} & \frac{\gamma_{2}^{2}+\xi^{2}}{\omega \mu_{0} \gamma_{2}} & \frac{\xi \eta}{\omega \mu_{0} \gamma_{2}}
\end{array}\right) \\
\times\left(\begin{array}{c}
\hat{E}_{1, x}^{-} \\
\hat{E}_{1, y}^{-} \\
\hat{E}_{2, x}^{+} \\
\hat{E}_{2, y}^{+}
\end{array}\right)=\left(\begin{array}{c}
n_{z} \xi / \omega \varepsilon_{s} \\
n_{z} \eta / \omega \varepsilon_{s} \\
n_{y} \\
-n_{x}
\end{array}\right) .
\end{gathered}
$$

Решение уравнения (42) имеет вид

$$
\begin{aligned}
\left(\begin{array}{c}
\hat{E}_{1, x}^{-} \\
\hat{E}_{1, y}^{-} \\
\hat{E}_{2, x}^{+} \\
\hat{E}_{2, y}^{+}
\end{array}\right)=Q\left[n_{x}\left(\begin{array}{c}
-\left(\gamma_{1} \gamma_{2}+\eta^{2}\right) \\
\xi \eta \\
-\left(\gamma_{1} \gamma_{2}+\eta^{2}\right) \\
\xi \eta
\end{array}\right)+n_{y}\left(\begin{array}{c}
\xi \eta \\
-\left(\gamma_{1} \gamma_{2}+\xi^{2}\right) \\
\xi \eta \\
-\left(\gamma_{1} \gamma_{2}+\xi^{2}\right)
\end{array}\right)\right. \\
\left.+n_{z}\left(\begin{array}{c}
-\xi \gamma_{1} \varepsilon_{2} / \varepsilon_{1,2} \\
-\eta \gamma_{1} \varepsilon_{2} / \varepsilon_{1,2} \\
\xi \gamma_{2} \varepsilon_{1} / \varepsilon_{1,2} \\
\eta \gamma_{2} \varepsilon_{1} / \varepsilon_{1,2}
\end{array}\right)\right]
\end{aligned}
$$

где

$$
Q=Q(\xi, \eta)=\frac{\omega \mu_{0}}{\left(\gamma_{1}+\gamma_{2}\right)\left(\gamma_{1} \gamma_{2}+\xi^{2}+\eta^{2}\right)}
$$

Для немагнитных сред в первом полупространстве (в области $j=1$ )

$$
\begin{gathered}
\left(\begin{array}{c}
\hat{E}_{1, x}^{-} \\
\hat{E}_{1, y}^{-}
\end{array}\right)=Q\left[n_{x}\left(\begin{array}{c}
-\left(\gamma_{1} \gamma_{2}+\eta^{2}\right) \\
\xi \eta
\end{array}\right)+n_{y}\left(\begin{array}{c}
\xi \eta \\
-\left(\gamma_{1} \gamma_{2}+\xi^{2}\right)
\end{array}\right)\right. \\
\left.+\frac{n_{z} \gamma_{1} \varepsilon_{2}}{\varepsilon_{s}}\left(\begin{array}{l}
-\xi \\
-\eta
\end{array}\right)\right]
\end{gathered}
$$

и во втором полупространстве (в области $j=2$ )

$$
\begin{aligned}
\left(\begin{array}{c}
\hat{E}_{2, x}^{+} \\
\hat{E}_{2, y}^{+}
\end{array}\right)= & Q\left[n_{x}\left(\begin{array}{c}
-\left(\gamma_{1} \gamma_{2}+\eta^{2}\right) \\
\xi \eta
\end{array}\right)+n_{y}\left(\begin{array}{c}
\xi \eta \\
-\left(\gamma_{1} \gamma_{2}+\xi^{2}\right)
\end{array}\right)\right. \\
& \left.+\frac{n_{z} \gamma_{2} \varepsilon_{1}}{\varepsilon_{s}}\left(\begin{array}{l}
\xi \\
\eta
\end{array}\right)\right] .
\end{aligned}
$$

Из (45), (46) для немагнитных сред находим поля в первом полупространстве (в области $j=1$ ) по формулам

$$
\begin{aligned}
& \mathbf{E}_{1}(x, y, z)=\frac{1}{(2 \pi)^{2}} \int_{-\infty}^{+\infty} \int_{-\infty}^{+\infty}\left(\begin{array}{cc}
1 & 0 \\
0 & 1 \\
\xi / \gamma_{1} & \eta / \gamma_{1}
\end{array}\right) \\
& \times\left(\begin{array}{c}
\hat{E}_{1, x}^{-} \\
\hat{E}_{1, y}^{-}
\end{array}\right) e^{-i \gamma_{1} z} e^{i(\xi x+\eta y)} d \xi d \eta \\
& \mathbf{B}_{1}(x, y, z)= \\
& =\frac{1}{(2 \pi)^{2}} \int_{-\infty}^{+\infty}\left(\begin{array}{cc}
-\left(\gamma_{1}^{2}+\xi^{2}\right) / \omega \gamma_{1} & -\xi \eta / \omega \gamma_{1} \\
-\eta & -\eta \omega \gamma_{1} \\
-\infty & \xi / \omega
\end{array}\right) \\
& \times\left(\begin{array}{c}
\hat{E}_{1, x}^{-} \\
\hat{E}_{1, y}^{-}
\end{array}\right) e^{-i \gamma_{1} z} e^{i(\xi x+\eta y)} d \xi d \eta,
\end{aligned}
$$


и во втором полупространстве (в области $j=2$ ) по формулам

$$
\begin{aligned}
& \mathbf{E}_{2}(x, y, z)=\frac{1}{(2 \pi)^{2}} \int_{-\infty}^{+\infty} \int_{-\infty}^{+\infty}\left(\begin{array}{cc}
1 & 0 \\
0 & 1 \\
-\xi / \gamma_{2} & -\eta / \gamma_{2}
\end{array}\right) \\
& \times\left(\begin{array}{c}
\hat{E}_{2, x}^{+} \\
\hat{E}_{2, y}^{+}
\end{array}\right) e^{-i \gamma_{2} z} e^{i(\xi x+\eta y)} d \xi d \eta, \\
& \mathbf{B}_{2}(x, y, z)=\int_{-\infty} \int_{-\infty}\left(\begin{array}{cc}
\left(\gamma_{2}^{2}+\xi \xi^{2}\right) / \omega \gamma_{2} & \xi \eta / \omega \gamma_{2} \\
-\eta / \omega & \xi / \omega
\end{array}\right) \\
& =\frac{1}{(2 \pi)^{2}} \int_{-\infty}^{+\infty}\left(\begin{array}{c}
-\xi \eta / \omega \gamma_{2} \\
\times\left(\begin{array}{c}
\hat{E}_{2, x}^{+} \\
\hat{E}_{2, y}^{+}
\end{array}\right) e^{-i \gamma_{2} z} e^{i(\xi x+\eta y)} d \xi d \eta .
\end{array}\right.
\end{aligned}
$$

\section{Элементарный горизонтальный диполь, расположенный на границе двух полупространств}

В качестве конкретного примера, который можно сравнить с известным решением [8], рассмотрим поле горизонтального единичного источника тока с параметрами $n_{x}=1, n_{y}=0, n_{z}=0$. Слово „горизонтальный“ мы будем использовать по историческим соображениям, данная задача возникла при рассмотрении горизонтальной дипольной антенны малого размера, расположенной на горизонтальной границе воздуха и земной поверхности. В данном рассмотрении .горизонтальный. означает параллельный плоской границе раздела. Подставляя в формулы (45), (46) указанные выше параметры горизонтального диполя $\left(n_{x}=1, n_{y}=0, n_{z}=0\right)$ и далее в $(47)-(50)$, для компоненты $E_{2, x}$ получим

$$
\begin{aligned}
& E_{2, x}(x, y, z)= \\
& =-\frac{\omega \mu_{0}}{(2 \pi)^{2}} \int_{-\infty}^{+\infty} \int_{-\infty}^{+\infty} \frac{\left(\gamma_{1} \gamma_{2}+\eta^{2}\right) e^{i \gamma_{2} z} e^{i(\xi x+\eta y)}}{\left(\gamma_{1}+\gamma_{2}\right)\left(\gamma_{1} \gamma_{2}+\xi^{2}+\eta^{2}\right)} d \xi d \eta .
\end{aligned}
$$

Переходя к полярным координатам в плоскостях $(x, y)$ и $(\xi, \eta)$ по формулам

$$
x=\rho \cos \varphi, \quad y=\rho \sin \varphi \quad \text { и } \quad \xi=\lambda \cos \vartheta, \quad \eta=\lambda \sin \vartheta
$$

получим $\gamma_{1}=\sqrt{k_{1}^{2}-\lambda^{2}}, \gamma_{2}=\sqrt{k_{2}^{2}-\lambda^{2}}$, а выражение для $E_{2, x}$ примет вид

$$
\begin{aligned}
& E_{2, x}(\rho, \varphi, z)= \\
& =-\frac{\omega \mu_{0}}{(2 \pi)^{2}} \int_{0}^{+\infty}\left(\int_{0}^{2 \pi} \frac{\left(\gamma_{1} \gamma_{2}+\lambda^{2} \sin ^{2} \vartheta\right) e^{i \gamma_{2} z} e^{i \rho \lambda \cos (\varphi-\vartheta)}}{\left(\gamma_{1}+\gamma_{2}\right)\left(\gamma_{1} \gamma_{2}+\lambda^{2}\right)} d \vartheta\right) \lambda d \lambda= \\
& -\frac{\omega \mu_{0}}{(2 \pi)^{2}} \int_{0}^{+\infty} \frac{e^{i \gamma_{2} z}}{\left(\gamma_{1}+\gamma_{2}\right)}\left(\int_{0}^{2 \pi} e^{i \rho \lambda \cos (\varphi-\vartheta)} d \vartheta\right) \lambda d \lambda \\
& +\frac{\omega \mu_{0}}{(2 \pi)^{2}} \int_{0}^{+\infty} \frac{\lambda^{2} e^{i \gamma_{2} z}}{\left(\gamma_{1}+\gamma_{2}\right)\left(\gamma_{1} \gamma_{2}+\lambda^{2}\right)}\left(\int_{0}^{2 \pi} e^{i \rho \lambda(\varphi-\vartheta)} \cos ^{2} \vartheta d \vartheta\right) \lambda d \lambda .
\end{aligned}
$$

Далее, используя интегральное представление функций Бесселя

$$
J_{n}(\rho \lambda)=\frac{i^{-n}}{2 \pi} \int_{0}^{2 \pi} e^{i \rho \lambda \cos \theta} e^{i n \theta} d \theta
$$

нетрудно получить следующие представления интегралов:

$$
\int_{0}^{2 \pi} e^{i \rho \lambda \cos (\varphi-\vartheta)} d \vartheta=2 \pi J_{0}(\rho \lambda)
$$

$$
\int_{0}^{2 \pi} e^{i \rho \lambda \cos (\varphi-\vartheta)} \cos ^{2} \vartheta d \vartheta=\pi\left(J_{0}(\rho \lambda)-J_{2}(\rho \lambda) \cos 2 \varphi\right) .
$$

Подставляя их, получим выражение для $E_{2, x}$ в цилиндрических координатах $(\rho, \varphi, z)$ :

$$
\begin{aligned}
& E_{2, x}(\rho, \varphi, z)=-\frac{\omega \mu_{0}}{4 \pi} \int_{0}^{+\infty}\left\{2 J_{0}(\rho \lambda)\right. \\
& \left.-\frac{\lambda^{2}\left(J_{0}(\rho \lambda)-J_{2}(\rho \lambda) \cos 2 \varphi\right)}{\left(\gamma_{1} \gamma_{2}+\lambda^{2}\right)}\right\} \frac{e^{i \gamma_{2} z}}{\left(\gamma_{1}+\gamma_{2}\right)} \lambda d \lambda .
\end{aligned}
$$

Аналогично получим выражение для $E_{1, x}$ :

$$
\begin{aligned}
& E_{1, x}(\rho, \varphi, z)=-\frac{\omega \mu_{0}}{4 \pi} \int_{0}^{+\infty}\left\{2 J_{0}(\rho \lambda)\right. \\
& \left.-\frac{\lambda^{2}\left(J_{0}(\rho \lambda)-J_{2}(\rho \lambda) \cos 2 \varphi\right)}{\left(\gamma_{1} \gamma_{2}+\lambda^{2}\right)}\right\} \frac{e^{-i \gamma_{1} z}}{\left(\gamma_{1}+\gamma_{2}\right)} \lambda d \lambda .
\end{aligned}
$$

Действуя таким же образом, для $E_{2, y}$ можно получить выражение

$$
E_{2, y}(x, y, z)=\frac{\omega \mu_{0}}{(2 \pi)^{2}} \int_{-\infty}^{+\infty} \int_{-\infty}^{+\infty} \frac{\xi \eta e^{i \gamma_{2} z} e^{i(\xi x+\eta y)}}{\left(\gamma_{1}+\gamma_{2}\right)\left(\gamma_{1} \gamma_{2}+\xi^{2}+\eta^{2}\right)} d \xi d \eta
$$


Снова переходя к полярным координатам в плоскостях $(x, y)$ и $(\xi, \eta)$, получим

$$
\begin{aligned}
& E_{2, y}(\rho, \varphi, z)=\frac{\omega \mu_{0}}{(2 \pi)^{2}} \int_{0}^{+\infty} \frac{\lambda^{2} e^{i \gamma_{2} z}}{\left(\gamma_{1}+\gamma_{2}\right)\left(\gamma_{1} \gamma_{2}+\lambda^{2}\right)} \\
& \times\left(\int_{0}^{2 \pi} \cos \vartheta \sin \vartheta e^{i \rho \lambda \cos (\varphi-\vartheta)} d \vartheta\right) \lambda d \lambda .
\end{aligned}
$$

Учитывая, что

$$
\begin{aligned}
& \int_{0}^{2 \pi} \sin \vartheta \cos \vartheta e^{i \rho \lambda \cos (\varphi-\vartheta)} d \vartheta= \\
& \quad=\pi \cdot \sin 2 \varphi\left(J_{0}(\rho \lambda)-\frac{2}{\rho \lambda} J_{1}(\rho \lambda)\right)
\end{aligned}
$$

получим

$$
\begin{aligned}
& E_{2, y}(\rho, \varphi, z)=\frac{\omega \mu_{0}}{4 \pi} \sin 2 \varphi \int_{0}^{+\infty} \frac{\lambda^{3} e^{i \gamma_{2} z}}{\left(\gamma_{1}+\gamma_{2}\right)\left(\gamma_{1} \gamma_{2}+\lambda^{2}\right)} \\
& \quad \times\left(J_{0}(\rho \lambda)-\frac{2}{\rho \lambda} J_{1}(\rho \lambda)\right) d \lambda
\end{aligned}
$$

Аналогично для $E_{1, y}$ получим

$$
\begin{aligned}
& E_{1, y}(\rho, \varphi, z)=\frac{\omega \mu_{0}}{4 \pi} \sin 2 \varphi \int_{0}^{+\infty} \frac{\lambda^{3} e^{-i \gamma_{1} z}}{\left(\gamma_{1}+\gamma_{2}\right)\left(\gamma_{1} \gamma_{2}+\lambda^{2}\right)} \\
& \quad \times\left(J_{0}(\rho \lambda)-\frac{2}{\rho \lambda} J_{1}(\rho \lambda)\right) d \lambda .
\end{aligned}
$$

Рассмотрим теперь компоненту $E_{2, z}$ :

$$
\begin{aligned}
& E_{2, z}(x, y, z)=\frac{\omega \mu_{0}}{(2 \pi)^{2}} \int_{-\infty}^{+\infty} \int_{-\infty}^{+\infty}\left(\frac{\xi}{\gamma_{2}} \hat{E}_{2, x}^{+}+\frac{\eta}{\gamma_{2}} \hat{E}_{2, y}^{+}\right) \\
& \times e^{i \gamma_{2} z} e^{i(\xi x+\eta y)} d \xi d \eta= \\
& =\frac{\omega \mu_{0}}{(2 \pi)^{2}} \int_{-\infty}^{+\infty} \int_{-\infty}^{+\infty} \frac{\xi \gamma_{1}}{\left(\gamma_{1}+\gamma_{2}\right)\left(\gamma_{1} \gamma_{2}+\xi^{2}+\eta^{2}\right)} e^{i \gamma_{2} z} e^{i(\xi x+\eta y)} d \xi d \eta .
\end{aligned}
$$

Переходя к полярным координатам, получим $E_{2, z}$ как функцию цилиндрических координат $(\rho, \varphi, z)$ в виде

$$
\begin{aligned}
& E_{2, z}(\rho, \varphi, z)=\frac{\omega \mu_{0}}{(2 \pi)^{2}} \int_{0}^{+\infty} \frac{\gamma_{1} \lambda^{2}}{\left(\gamma_{1}+\gamma_{2}\right)\left(\gamma_{1} \gamma_{2}+\lambda^{2}\right)} e^{i \gamma_{2} z} \\
& \quad \times\left(\int_{0}^{2 \pi} e^{i \rho \lambda \cos (\varphi-\vartheta)} \cos \vartheta d \vartheta\right) d \lambda
\end{aligned}
$$

Учитывая (53), получим интегральное представление

$$
\int_{0}^{2 \pi} e^{i \rho \lambda \cos (\varphi-\vartheta)} \cos \vartheta d \vartheta=2 \pi i J_{1}(\rho \lambda) \cos \varphi,
$$

и для $E_{2, z}$ следующее выражение:

$$
E_{2, z}(\rho, \varphi, z)=\frac{i \omega \mu_{0} \cos \varphi}{2 \pi} \int_{0}^{+\infty} \frac{\gamma_{1} \lambda^{2} J_{1}(\rho \lambda) e^{i \gamma_{2} z}}{\left(\gamma_{1}+\gamma_{2}\right)\left(\gamma_{1} \gamma_{2}+\lambda^{2}\right)} d \lambda
$$

Аналогично для $E_{1, z}$ получим выражение

$$
E_{1, z}(\rho, \varphi, z)=-\frac{i \omega \mu_{0} \cos \varphi}{2 \pi} \int_{0}^{+\infty} \frac{\gamma_{2} \lambda^{2} J_{1}(\rho \lambda) e^{-i \gamma_{1} z}}{\left(\gamma_{1}+\gamma_{2}\right)\left(\gamma_{1} \gamma_{2}+\lambda^{2}\right)} d \lambda
$$

Полученные выражения в точности приводятся к решениям для горизонтального диполя [8].

\section{Излучение элементарного горизонтального диполя, расположенного на границе металлической пленки, нанесенной на поверхность призмы}

Рассмотрим теперь задачу излучения элементарного источника, расположенного на свободной границе пленки (рис. 5) в схеме Кречмана. Поляризация источника вдоль оси $X\left(n_{x}=1, n_{y}=0, n_{z}=0\right)$. Толщина пленки равна $d$. Индекс 1 соответствует полупространству материала призмы, 2 - пленке, а 3 - свободному полупространству над пленкой. Тогда $N=2, z_{d}=z_{2}=d$, $\mathbf{H}_{R}=\mathbf{T}_{3}, \mathbf{H}_{L}=\left(\mathbf{T}_{1} \times \mathbf{T}_{2}\right)^{-1}$ и уравнение (33) примет вид

$$
\mathbf{T}_{3} \times \hat{\mathfrak{E}}_{3}=\left(\mathbf{T}_{1} \times \mathbf{T}_{2}\right)^{-1} \times \hat{\mathfrak{E}}_{1}+\mathbf{V},
$$

где матрицы выражаются следующими формулами:

$$
\begin{gathered}
\mathbf{T}_{1}=\left(\begin{array}{cc}
\mathbf{I} & \mathbf{I} \\
\mathbf{G}_{1} & -\mathbf{G}_{1}
\end{array}\right)^{-1}, \\
\mathbf{T}_{2}=\left(\begin{array}{cc}
\mathbf{I} & e^{i \gamma_{2} d} \mathbf{I} \\
\mathbf{G}_{2} & -e^{-i \gamma_{2} d} \mathbf{G}_{2}
\end{array}\right)\left(\begin{array}{cc}
e^{i \gamma_{2} d} \mathbf{I} & \mathbf{I} \\
e^{i \gamma_{2} d} \mathbf{G}_{2} & -\mathbf{G}_{2}
\end{array}\right)^{-1}, \\
\mathbf{T}_{3}=\left(\begin{array}{cc}
\mathbf{I} & \mathbf{I} \\
\mathbf{G}_{3} & -\mathbf{G}_{3}
\end{array}\right),
\end{gathered}
$$

a вектор-столбец стороннего тока равен $\mathbf{V}=(0 ; 0 ; 0 ;-1)^{T}$.

Учитывая, что $\left(\mathbf{T}_{1} \times \mathbf{T}_{2}\right)^{-1}=\mathbf{T}_{2}^{-1} \times \mathbf{T}_{1}^{-1}$, получим

$$
\begin{aligned}
& \mathbf{H}_{L}=\left(\mathbf{T}_{1} \times \mathbf{T}_{2}\right)^{-1}=\mathbf{T}_{2}^{-1} \times \mathbf{T}_{1}^{-1}= \\
& =\left(\begin{array}{cc}
e^{i \gamma_{2} d} \mathbf{I} & \mathbf{I} \\
e^{i \gamma_{2} d} \mathbf{G}_{2} & -\mathbf{G}_{2}
\end{array}\right)\left(\begin{array}{cc}
\mathbf{I} & e^{i \gamma_{2} d} \mathbf{I} \\
\mathbf{G}_{2} & -e^{i \gamma_{2} d} \mathbf{G}_{2}
\end{array}\right)^{-1} \times\left(\begin{array}{cc}
\mathbf{I} & \mathbf{I} \\
\mathbf{G}_{1} & -\mathbf{G}_{1}
\end{array}\right) .
\end{aligned}
$$




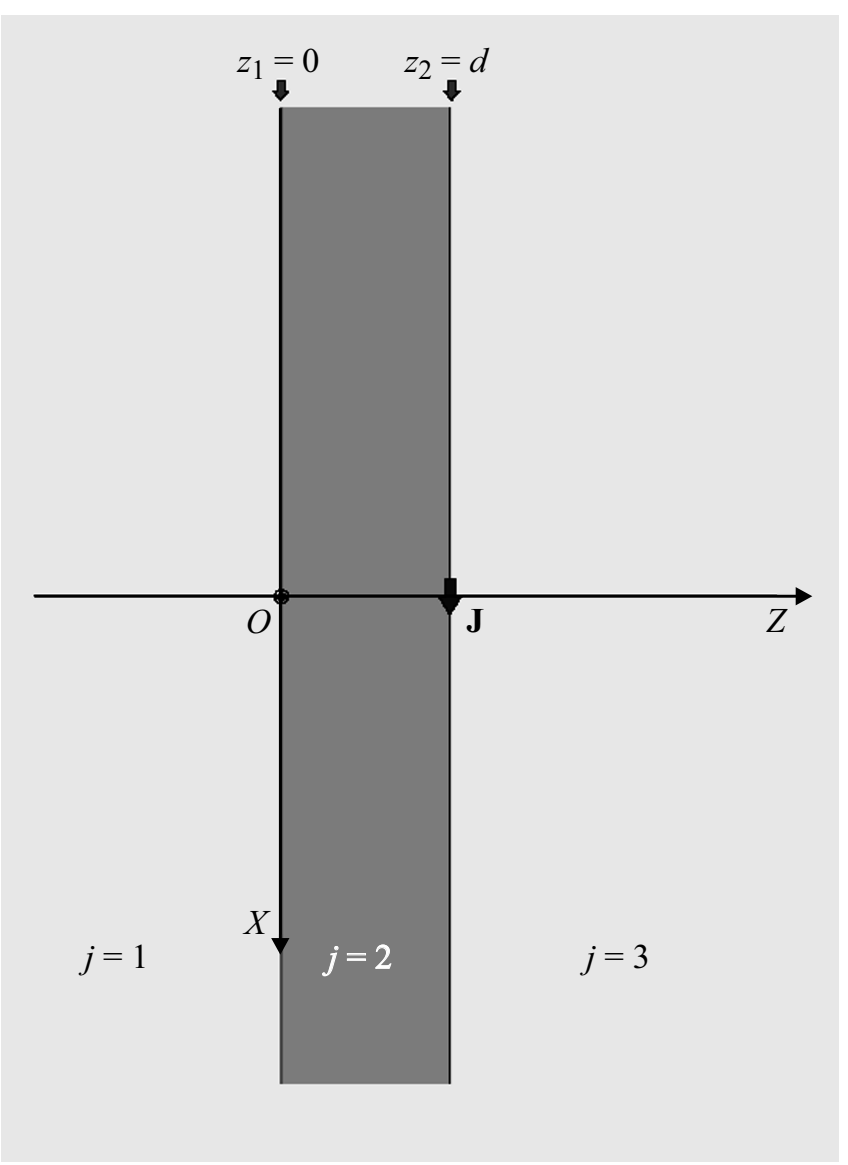

Рис. 5. Геометрия задачи излучения элементарного источника, расположенного на свободной границе пленки в схеме Кречмана.

Вводя вектор-столбец $\hat{\mathfrak{E}}_{\text {out }}=\left(\hat{E}_{1, x}^{-} ; \hat{E}_{1, y}^{-} ; \hat{E}_{3, x}^{+} ; \hat{E}_{3, y}^{+}\right)^{T}$, уравнение (35) для данной задачи примет вид

$$
\left(\begin{array}{cc}
-\mathbf{H}_{L B} & \mathbf{I} \\
-\mathbf{H}_{L D} & \mathbf{G}_{3}
\end{array}\right) \times \hat{\mathfrak{E}}_{\text {out }}=\mathbf{V}
$$

Решая линейное уравнение (59), найдем $\hat{E}_{1, x}^{-}, \hat{E}_{1, y}^{-}$и $\hat{E}_{3, x}^{+}, \hat{E}_{3, y}^{+}$и далее по формулам (36)-(39) при $N=2$ получим уходящую волну из плоскослоистой структуры в призму (в область 1) и свободное пространство (область 3) над пленкой (в направлениях $z \rightarrow \pm \infty$ от границ пленки).

Снова будем считать, что среды немагнитные и $\mu_{1}=\mu_{2}=\mu_{3}=\mu_{0}$. Тогда, учитывая $k_{j}^{2}=\varepsilon_{j} \mu_{0} \omega^{2}$, выражения для подматриц $\mathbf{H}_{L B}$ и $\mathbf{H}_{L D}$ можно получить в явном виде.

Подматрица $\mathbf{H}_{L B}$ :

$$
\begin{gathered}
\left(\mathbf{H}_{L B}\right)_{0,0}=\cos \left(\gamma_{2} d\right)+i\left(\left(\frac{\varepsilon_{1}-\varepsilon_{2}}{\varepsilon_{2}}\right) \frac{\xi^{2}}{\gamma_{1}}-\gamma_{1}\right) \frac{\sin \left(\gamma_{2} d\right)}{\gamma_{2}} \\
\left(\mathbf{H}_{L B}\right)_{0,1}=i \xi \eta \frac{\left(\varepsilon_{1}-\varepsilon_{2}\right)}{\varepsilon_{2}} \frac{\sin \left(\gamma_{2} d\right)}{\gamma_{1} \gamma_{2}}
\end{gathered}
$$

$$
\begin{gathered}
\left(\mathbf{H}_{L B}\right)_{1,0}=i \xi \eta \frac{\left(\varepsilon_{1}-\varepsilon_{2}\right)}{\varepsilon_{2}} \frac{\sin \left(\gamma_{2} d\right)}{\gamma_{1} \gamma_{2}}, \\
\left(\mathbf{H}_{L B}\right)_{1,1}=\cos \left(\gamma_{2} d\right)+i\left(\left(\frac{\varepsilon_{1}-\varepsilon_{2}}{\varepsilon_{2}}\right) \frac{\eta^{2}}{\gamma_{1}}-\gamma_{1}\right) \frac{\sin \left(\gamma_{2} d\right)}{\gamma_{2}},
\end{gathered}
$$

подматрица $\mathbf{H}_{L D}$ :

$$
\begin{gathered}
\left(\mathbf{H}_{L D}\right)_{0,0}=\frac{\xi \eta}{\mu_{0} \omega}\left(\frac{\cos \left(\gamma_{2} d\right)}{\gamma_{1}}-i \frac{\sin \left(\gamma_{2} d\right)}{\gamma_{2}}\right), \\
\left(\mathbf{H}_{L D}\right)_{0,1}=\frac{\left(\gamma_{1}^{2}+\eta^{2}\right)}{\gamma_{1} \mu_{0} \omega} \cos \left(\gamma_{2} d\right) \\
-i \frac{\gamma_{2}^{2}+\eta^{2}}{\gamma_{2} \mu_{0} \omega} \sin \left(\gamma_{2} d\right), \\
\left(\mathbf{H}_{L D}\right)_{1,0}=-\frac{\left(\gamma_{1}^{2}+\xi^{2}\right)}{\gamma_{1} \mu_{0} \omega} \cos \left(\gamma_{2} d\right) \\
+i \frac{\gamma_{2}^{2}+\xi^{2}}{\gamma_{2} \mu_{0} \omega} \sin \left(\gamma_{2} d\right), \\
\left(\mathbf{H}_{L D}\right)_{1,1}=-\frac{\xi \eta}{\mu_{0} \omega}\left(\frac{\cos \left(\gamma_{2} d\right)}{\gamma_{1}}-i \frac{\sin \left(\gamma_{2} d\right)}{\gamma_{2}}\right)
\end{gathered}
$$

Решив уравнение (59), найдем следующие аналитические выражения для $\hat{E}_{1, x}^{-}, \hat{E}_{1, y}^{-}, \hat{E}_{1, z}^{-}$:

$$
\hat{E}_{1, x}=\frac{\left(a_{1 x}+d_{1 x} \eta^{2}\right)}{\omega \mu_{0} D} \cos \left(\gamma_{2} d\right)+i \frac{\left(b_{1 x}+e_{1 x} \eta^{2}\right)}{\omega \mu_{0} D} \sin \left(\gamma_{2} d\right) \text {, }
$$

где

$$
\begin{gathered}
a_{1 x}=-\left(\gamma_{1}+\gamma_{3}\right), \quad d_{1 x}=-\left(\gamma_{1}+\gamma_{3}\right) / \gamma_{1} \gamma_{3}, \\
b_{1 x}=\left(\gamma_{1} \gamma_{3}+\gamma_{2}^{2}\right) / \gamma_{2}, \quad e_{1 x}=\frac{\left(\gamma_{3}+\gamma_{1}\right)}{\gamma_{2} \gamma_{3}}+\frac{\left(\varepsilon_{2}-\varepsilon_{1}\right) k_{3}^{2}}{\varepsilon_{2} \gamma_{1} \gamma_{2} \gamma_{3}}, \\
\hat{E}_{1, y}^{-}=\frac{1}{\omega \mu_{0} D}\left(a_{1 y} \cos \left(\gamma_{2} d\right)+i b_{1 y} \sin \left(\gamma_{2} d\right)\right) \xi \eta ; \\
\text { где } a_{1 y}=\left(\gamma_{1}+\gamma_{3}\right) / \gamma_{1} \gamma_{3}, \\
b_{1 y}=\frac{1}{\gamma_{1} \gamma_{2} \gamma_{3}}\left(\frac{\left(\varepsilon_{1}-\varepsilon_{2}\right) k_{3}^{2}}{\varepsilon_{2}}-\gamma_{1}\left(\gamma_{1}+\gamma_{3}\right)\right) . \\
\hat{E}_{1, z}^{-}=\xi \hat{E}_{1, x}^{-} / \gamma_{1}+\eta \hat{E}_{1, y}^{-} / \gamma_{1}
\end{gathered}
$$

и $\hat{E}_{3, x}^{+}, \hat{E}_{3, y}^{+}, \hat{E}_{3, z}^{+}$:

$$
\begin{aligned}
\hat{E}_{3, x}^{+}= & \frac{\left(a_{3 x}+d_{3 x} \eta^{2}\right)}{\omega \mu_{0} D} \cos ^{2}\left(\gamma_{2} d\right) \\
& +\frac{\left(b_{3 x}+e_{3 x} \eta^{2}\right)}{\omega \mu_{0} D} \sin ^{2}\left(\gamma_{2} d\right) \\
& +i \frac{\left(c_{3 x}+f_{3 x} \eta^{2}\right)}{\omega \mu_{0} D} \cos \left(\gamma_{2} d\right) \sin \left(\gamma_{2} d\right) ;
\end{aligned}
$$

где

$$
\begin{gathered}
a_{3 x}=-\left(\gamma_{1}+\gamma_{3}\right), \\
d_{3 x}=-\left(\gamma_{1}+\gamma_{3}\right) / \gamma_{1} \gamma_{3},
\end{gathered}
$$




$$
\begin{gathered}
b_{3 x}=\left[\gamma_{1} \gamma_{3}\left(k_{3}^{2}-\gamma_{3}^{2}\right)+\gamma_{3} \gamma_{1}^{3}+\gamma_{2}^{2} k_{1}^{2}\right] /\left(\gamma_{1} k_{2}^{2}\right), \\
e_{3 x}=\left[\gamma_{3} \gamma_{1}^{2}\left(k_{2}^{2}+\gamma_{2}^{2}\right)-\gamma_{3} \gamma_{2}^{4}+k_{1}^{2} \gamma_{1} \gamma_{2}^{2}\right] /\left(k_{2}^{2} \gamma_{1} \gamma_{2}^{2} \gamma_{3}\right), \\
c_{3 x}=\frac{\gamma_{3}}{\gamma_{1} \gamma_{2} k_{2}^{2}}\left(\left(\gamma_{2}^{2}+\gamma_{1}^{2}\right)\left(k_{3}^{2}-\gamma_{3}^{2}\right)+2 \gamma_{1}^{2} \gamma_{2}^{2}\right) \\
\quad+\frac{1}{\gamma_{2} k_{2}^{2}}\left(\gamma_{2}^{4}+\gamma_{1}^{2} \gamma_{2}^{2}+2 \gamma_{2}^{2}\left(k_{2}^{2}-\gamma_{2}^{2}\right)\right), \\
f_{3 x=} \frac{1}{\gamma_{1} \gamma_{2} \gamma_{3} k_{2}^{2}}\left(k_{3}^{2}\left(\gamma_{2}^{2}+\gamma_{1}^{2}\right)+2 \gamma_{1}^{2} \gamma_{2}^{2}-\gamma_{3}^{2}\left(\gamma_{2}^{2}+\gamma_{1}^{2}\right)\right) \\
+\frac{1}{\gamma_{2} k_{2}^{2}}\left(2 k_{2}^{2}+\gamma_{1}^{2}-\gamma_{2}^{2}\right) . \\
\hat{E}_{3, y}^{+}=\frac{1}{\omega \mu_{0} D}\left(a_{3 y} \cos ^{2}\left(\gamma_{2} d\right)+b_{3 y} \sin ^{2}\left(\gamma_{2} d\right)\right. \\
\left.+i c_{3 y} \cos \left(\gamma_{2} d\right) \sin \left(\gamma_{2} d\right)\right) \xi \eta,
\end{gathered}
$$

где

$$
\begin{gathered}
a_{3 y}=\left(\gamma_{1}+\gamma_{3}\right) / \gamma_{1} \gamma_{3}, \\
b_{3 y}=\frac{\left(\varepsilon_{1}-\varepsilon_{2}\right)}{\varepsilon_{2}} \frac{\left(\gamma_{1}\left(k_{3}^{2}-\gamma_{3}^{2}\right)-\gamma_{2}^{2} \gamma_{3}\right)}{\gamma_{1} \gamma_{2}^{2} \gamma_{3}}-\frac{\gamma_{1}\left(\gamma_{1}+\gamma_{3}\right)}{\gamma_{2}^{2} \gamma_{3}}, \\
c_{3 y}=\frac{\left(\varepsilon_{1}-\varepsilon_{2}\right)}{\varepsilon_{2}} \frac{\left(k_{3}^{2}-\gamma_{3}\left(\gamma_{1}+\gamma_{3}\right)\right)}{\gamma_{1} \gamma_{2} \gamma_{3}}-\frac{2\left(\gamma_{1}+\gamma_{3}\right)}{\gamma_{2} \gamma_{3}}, \\
\hat{E}_{3, z}^{+}=-\xi \hat{E}_{3, x}^{+} / \gamma_{3}-\eta \hat{E}_{3, y}^{+} / \gamma_{3} .
\end{gathered}
$$

В формулах $(60)-(65)$ функция $D(\xi, \eta)$ есть детерминант матрицы уравнения (59), представляющийся следующим аналитическим выражением:

$$
\begin{aligned}
& D(\xi, \eta)=\frac{1}{\omega^{2} \mu_{0}^{2}} \frac{\left(\gamma_{1}+\gamma_{3}\right)^{2}\left(\gamma_{1} \gamma_{3}+\left(\xi^{2}+\eta^{2}\right)\right)}{\gamma_{1} \gamma_{3}} \cos ^{2}\left(\gamma_{2} d\right) \\
& -\frac{1}{\omega^{2} \mu_{0}^{2}} \frac{\left(\gamma_{1} \gamma_{2}+\gamma_{2}^{2}\right)^{2}}{\gamma_{1} \gamma_{3} \gamma_{2}^{2} k_{2}^{2}}\left(\left(\xi^{2}+\eta^{2}\right)^{2}\right. \\
& \left.+\frac{\gamma_{2}^{2}\left(\gamma_{1}+\gamma_{3}\right)^{2}}{\gamma_{1} \gamma_{3}+\gamma_{2}^{2}}\left(\xi^{2}+\eta^{2}\right)+\gamma_{1} \gamma_{2}^{2} \gamma_{3}\right) \sin ^{2}\left(\gamma_{2} d\right) \\
& -i \frac{\left(\gamma_{1}+\gamma_{3}\right)}{\omega^{2} \mu_{0}^{2} \gamma_{1} \gamma_{2} \gamma_{3} k_{2}^{2}}\left(2\left(\gamma_{1} \gamma_{3}+\gamma_{2}^{2}\right)\left(\gamma_{1} \gamma_{3} \gamma_{2}^{2}+\left(\xi^{2}+\eta^{2}\right)^{2}\right)\right. \\
& +\left(\gamma_{1}^{2} \gamma_{3}^{2}+\gamma_{1}^{2} \gamma_{2}^{2}+4 \gamma_{1} \gamma_{3} \gamma_{2}^{2}+\gamma_{3}^{2} \gamma_{2}^{2}+\gamma_{2}^{4}\right) \\
& \left.\times\left(\xi^{2}+\eta^{2}\right)\right) \cos \left(\gamma_{2} d\right) \sin \left(\gamma_{2} d\right) .
\end{aligned}
$$

Естественно, что полученные выражения (60)-(65) переходят в соответствующие выражения для диполя на границе двух полупространств, когда толщина пленки $d$ стремится к нулю.

Аналитические выражения (60)-(65) имеют первостепенное значение, так как позволяют получить выражение для электрических полей в полупространствах через одномерные интегралы, что значительно ускоряет счет и повышает точность вычислений. Действительно, если перейти к полярным координатам в плоскостях $(x, y)$ и $(\xi, \eta)$ по формулам $x=\rho \cos \varphi, y=\rho \sin \varphi$ и $\xi=\lambda \cos \vartheta$, $\eta=\lambda \sin \vartheta$, получим, что величины $\gamma_{1}=\sqrt{k_{1}^{2}-\lambda^{2}}$, $\gamma_{2}=\sqrt{k_{2}^{2}-\lambda^{2}}, \gamma_{3}=\sqrt{k_{3}^{2}-\lambda}$, выделенные выше множители и детерминант $\mathfrak{D}(\lambda)=D(\lambda \cos \vartheta, \lambda \sin \vartheta)$ являются функциями только $\lambda$ и не зависят от $\vartheta$. Поэтому выражения для соответствующих полей, с вычислительной точки зрения, имеют такой же вид, что и выражения (51), (55), (57), и с помощью интегрального представления функций Бесселя (53) приводятся к одномерным интегралам.

Рассмотрим электрические поля в полупространствах (в областях с номером $j=1$ и $j=3$ ). Сначала рассмотрим (см. представление (36)) в полупространстве c $j=1$ :

$$
E_{1, x}(x, y, z)=\frac{1}{(2 \pi)^{2}} \int_{-\infty}^{+\infty} \int_{-\infty}^{+\infty} \hat{E}_{1, x}^{-} e^{-i \gamma_{1} z} e^{i(\xi x+\eta y)} d \xi d \eta .
$$

Подставим выражение $(60)$ для $\hat{E}_{1, x}^{-}$и перейдем к полярным координатам (52), получим

$$
\begin{aligned}
& E_{1, x}(\rho, \varphi, z)=\frac{1}{(2 \pi)^{2}} \int_{0}^{+\infty}\left(\int_{0}^{2 \pi}\left(Q_{1 x}(y)+R_{1 x}(\gamma) \lambda^{2} \sin ^{2} \vartheta\right)\right. \\
& \left.\times e^{-i \gamma_{1} z} e^{i \rho \lambda \cos (\varphi-\vartheta)} d \vartheta\right) \lambda d \lambda \\
& =\frac{1}{(2 \pi)^{2}} \int_{0}^{+\infty}\left(Q_{1 x}(\gamma)+R_{1 x}(\gamma) \lambda^{2}\right) \lambda e^{-i \gamma_{1} z} \\
& \times\left(\int_{0}^{2 \pi} e^{i \rho \lambda \cos (\varphi-\vartheta)} d \vartheta\right) d \lambda-\frac{1}{(2 \pi)^{2}} \int_{0}^{+\infty} R_{1 x}(\gamma) \lambda^{3} e^{-i \gamma_{1} z} \\
& \times\left(\int_{0}^{2 \pi} \cos ^{2} \vartheta e^{i \rho \lambda \cos (\varphi-\vartheta)} d \vartheta\right) d \lambda,
\end{aligned}
$$

где введены следующие (зависящие только от $\lambda$ ) функции:

$$
\begin{aligned}
Q_{1 x}(\gamma) & =\frac{1}{\omega \mu_{0} \mathfrak{D}(\lambda)}\left(a_{1 x} \cos \left(\gamma_{2} d\right)+i b_{1 x} \sin \left(\gamma_{2} d\right)\right), \\
R_{1 x}(\gamma) & =\frac{1}{\omega \mu_{0} \mathfrak{D}(\lambda)}\left(d_{1 x} \cos \left(\gamma_{2} d\right)+i e_{1 x} \sin \left(\gamma_{2} d\right)\right) .
\end{aligned}
$$

Подставляя выражения (54), получим

$$
\begin{aligned}
& E_{1, x}(\rho, \varphi, z)=\frac{1}{4 \pi} \int_{0}^{+\infty}\left(2 Q_{1 x}(\gamma)+R_{1 x}(\gamma) \lambda^{2}\right) \\
& \times \lambda e^{-i \gamma_{1} z} J_{0}(\rho \lambda) d \lambda+\frac{\cos 2 \varphi}{4 \pi} \int_{0}^{+\infty} R_{1 x}(\gamma) \lambda^{3} J_{2}(\rho \lambda) e^{-i \gamma_{1} z} d \lambda,
\end{aligned}
$$


Аналогично для $y$-компоненты. Из (36) и (62), используя

$$
E_{1, y}(x, y, z)=\frac{1}{(2 \pi)^{2}} \int_{-\infty}^{+\infty} \int_{-\infty}^{+\infty} \hat{E}_{1, y}^{-} e^{-i \gamma_{1} z} e^{i(\xi x+\eta y)} d \xi d \eta,
$$

и переходя к полярным координатам, получим

$$
\begin{aligned}
& E_{1, y}(\rho, \varphi, z)=\frac{1}{(2 \pi)^{2}} \int_{0}^{+\infty}\left(\int _ { 0 } ^ { 2 \pi } \left(Q_{1 y}(\gamma) \lambda^{2} \cos \vartheta \sin \vartheta\right.\right. \\
& \left.\times e^{-i \gamma_{1} \lambda \cos (\varphi-\vartheta)} d \vartheta\right) \lambda d \lambda=\frac{1}{(2 \pi)^{2}} \int_{0}^{+\infty} \lambda^{3} Q_{1 y}(\gamma) e^{-i \gamma_{1} z} \\
& \times\left(\int_{0}^{2 \pi} \cos \vartheta \sin \vartheta e^{i \gamma_{1} \lambda \cos (\varphi-\vartheta)} d \vartheta\right) d \lambda,
\end{aligned}
$$

где введена функция от $\lambda$

$$
Q_{1 y}(\gamma)=\frac{1}{\omega \mu_{0} \mathfrak{D}(\lambda)}\left(a_{1 y} \cos \left(\gamma_{2} d\right)+i b_{1 y} \sin \left(\gamma_{2} d\right)\right) .
$$

Учитывая интегральное представление (56), получим для $E_{1, y}$ выражение

$$
\begin{aligned}
& E_{1, y}(\rho, \varphi, z)=\frac{\sin 2 \varphi}{4 \pi} \int_{0}^{+\infty} \lambda^{3} Q_{1 y}(\gamma) \\
& \times\left(J_{0}(\rho \lambda)-\frac{2}{\rho \lambda} J_{1}(\rho \lambda)\right) e^{-i \gamma_{1} z} d \lambda .
\end{aligned}
$$

Оставшуюся компоненту $E_{1, z}$ в первом полупространстве из (36) запишем в виде

$$
\begin{aligned}
E_{1, z}(x, y, z)= & \frac{1}{(2 \pi)^{2}} \int_{-\infty}^{\infty} \int_{-\infty}^{\infty}\left(\xi \hat{E}_{1, x}^{-} / \gamma_{1}+\eta \hat{E}_{1, y}^{-} / \gamma_{1}\right) \\
& \times e^{-i \gamma_{1} z} e^{i(\xi x+\eta y)} d \xi d \eta,
\end{aligned}
$$

и, подставляя выражения для $\hat{E}_{1, x}^{-}$и $\hat{E}_{1, y}^{-}$, по формулам (60) и (61) после перехода к полярным координатам получим

$$
\begin{aligned}
& E_{1, z}(\rho, \varphi, z)=\frac{1}{(2 \pi)^{2}} \int_{0}^{+\infty} \frac{Q_{1 x}(\gamma) \lambda^{2} e^{i \gamma_{1} z}}{\gamma_{1}} \\
& \times\left(\int_{0}^{2 \pi} \cos \vartheta e^{i \rho \lambda \cos (\varphi-\vartheta)} d \vartheta\right) d \lambda \\
& +\frac{1}{(2 \pi)^{2}} \int_{0}^{+\infty} \frac{\left(R_{1 x}(\gamma)+Q_{1 y}(\gamma)\right) \lambda^{4}}{\gamma_{1}} e^{i \gamma_{1} z} \\
& \times\left(\int_{0}^{2 \pi} \cos \vartheta \sin ^{2} \vartheta e^{i \rho \lambda \cos (\varphi-\vartheta)} d \vartheta\right) d \lambda .
\end{aligned}
$$

Получая из (53) представления

$$
\int_{0}^{2 \pi} \cos \vartheta e^{i \rho \lambda \cos (\varphi-\vartheta)} d \vartheta=2 \pi i \cos \varphi J_{1}(\rho \lambda)
$$

$$
\int_{0}^{2 \pi} \cos \vartheta \sin ^{2} \vartheta e^{i \rho \lambda \cos (\varphi-\vartheta)} d \vartheta=i 2 \pi \cos \varphi
$$

$$
\times\left(\sin ^{2} \varphi J_{1}(\rho \lambda)+\left(1-4 \sin ^{2} \varphi\right) \frac{J_{2}(\rho \lambda)}{\rho \lambda}\right)
$$

находим

$$
E_{1, z}(\rho, \varphi, z)=\frac{i \cos \varphi}{2 \pi} \int_{0}^{+\infty} \frac{Q_{1 x}(\gamma) \lambda^{2} e^{-i \gamma_{1} z}}{\gamma_{1}} J_{1}(\rho \lambda) d \lambda
$$

$$
\begin{aligned}
& +\frac{i \cos \varphi \sin ^{2} \varphi}{2 \pi} \int_{0}^{+\infty} \frac{\left(R_{1 x}(\gamma)+Q_{1 y}(\gamma)\right) \lambda^{4} J_{1}(\rho \lambda)}{\gamma_{1}} e^{-i \gamma_{1} z} d \lambda \\
& +\frac{i \cos \varphi\left(1-4 \sin ^{2} \varphi\right)}{2 \pi}
\end{aligned}
$$

$$
\times \int_{0}^{+\infty} \frac{\left(R_{1 x}(\gamma)+Q_{1 y}(\gamma)\right) \lambda^{3} J_{2}(\rho \lambda)}{\rho \gamma_{1}} e^{-i \gamma_{1} z} d \lambda
$$

Теперь рассмотрим электрическое поле в полупространстве (с номером $j=3$ ). Составляющую по оси $x$ получим из (38) в виде

$$
E_{3, x}(x, y, z)=\frac{1}{(2 \pi)^{2}} \int_{-\infty}^{+\infty} \int_{-\infty}^{+\infty} \hat{E}_{3, x}^{+} e^{i \gamma_{3}(z-d)} e^{i(\xi x+\eta y)} d \xi d \eta
$$

Преобразуя выражение (63), представим его в виде

$$
\begin{aligned}
& \hat{E}_{3, x}^{+}=\frac{1}{\omega \mu_{0} D}\left[a_{3 x} \cos ^{2}\left(\gamma_{2} d\right)+b_{3 x} \sin ^{2}\left(\gamma_{2} d\right)\right. \\
& +i c_{3 x} \cos \left(\gamma_{2} d\right) \sin \left(\gamma_{2} d\right)+\left(d_{3 x} \cos ^{2}\left(\gamma_{2} d\right)\right. \\
& \left.\left.+e_{3 x} \sin ^{2}\left(\gamma_{2} d\right)+i f_{3 x} \cos \left(\gamma_{2} d\right) \sin \left(\gamma_{2} d\right)\right) \eta^{2}\right] .
\end{aligned}
$$


Тогда, переходя к полярным координатам (52), получим

$$
\begin{aligned}
& E_{3, x}(\rho \varphi, z)=\frac{1}{(2 \pi)^{2}} \int_{0}^{+\infty}\left(\int_{0}^{2 \pi}\left(Q_{3 x}(\gamma)+R_{3 x}(\gamma) \lambda^{2} \sin ^{2} \vartheta\right)\right. \\
& \left.\times e^{i \gamma_{3}(z-d)} e^{i \rho \lambda \cos (\varphi-\vartheta)} d \vartheta\right) \lambda d \lambda= \\
& =\frac{1}{(2 \pi)^{2}} \int_{0}^{+\infty}\left(Q_{3 x}(\gamma)+\lambda^{2} R_{3 x}(\gamma)\right) e^{i \gamma_{3}(z-d)} \\
& \times\left(\int_{0}^{2 \pi} e^{i \rho \lambda \cos (\varphi-\vartheta)} d \vartheta\right) \lambda d \lambda-\frac{1}{(2 \pi)^{2}} \int_{0}^{+\infty}\left(\lambda^{2} R_{3 x}(\gamma)\right) \\
& \times e^{i \gamma_{3}(z-d)}\left(\int_{0}^{2 \pi} \cos ^{2} \vartheta e^{i \rho \lambda(\varphi-\vartheta)} d \vartheta\right) \lambda d \lambda
\end{aligned}
$$

где введены следующие, зависящие только от $\lambda$, функции:

$$
\begin{aligned}
Q_{3 x}(\gamma)= & \frac{1}{\omega \mu_{0} \mathfrak{D}(\lambda)}\left(a_{3 x} \cos ^{2}\left(\gamma_{2} d\right)+b_{3 x} \sin ^{2}\left(\gamma_{2} d\right)\right. \\
& \left.+i c_{3 x} \cos \left(\gamma_{2} d\right) \sin \left(\gamma_{2} d\right)\right), \\
R_{3 x}(\gamma)= & \frac{1}{\omega \mu_{0} \mathfrak{D}(\lambda)}\left(d_{3 x} \cos ^{2}\left(\gamma_{2} d\right)+e_{3 x} \sin ^{2}\left(\gamma_{2} d\right)\right. \\
& \left.+i f_{3 x} \cos \left(\gamma_{2} d\right) \sin \left(\gamma_{2} d\right)\right),
\end{aligned}
$$

Подставляя выражения (54), получим

$$
\begin{aligned}
& E_{3, y}(\rho, \varphi, z)=\frac{1}{4 \pi} \int_{0}^{+\infty}\left(2 Q_{3 x}(\gamma)+\lambda^{2} R_{3 x}(\gamma)\right) J_{0}(\rho \lambda) \\
& \times e^{i \gamma_{3}(z-d)} \lambda d \lambda+\frac{\cos 2 \varphi}{4 \pi} \int_{0}^{+\infty} \lambda^{3} R_{3 x}(\gamma) J_{2}(\rho \lambda) e^{i \gamma_{3}(z-d)} d \lambda .
\end{aligned}
$$

Аналогично в представлении

$$
E_{3, y}(x, y, z)=(2 \pi) \int_{-\infty}^{+\infty} \int_{-\infty}^{+\infty} \hat{E}_{3, y}^{+} e^{i \gamma_{3}(z-d)} e^{i(\xi x+\eta y)} d \xi d \eta
$$

запишем $\hat{E}_{3, y}^{+}$в виде $(63)$ и перейдем к полярным координатам:

$$
\begin{aligned}
& E_{3, y}(\rho, \omega, z)=\frac{1}{(2 \pi)^{2}} \int_{0}^{+\infty}\left(\int_{0}^{2 \pi} Q_{3 y}(\gamma) \lambda^{2} \cos \vartheta \sin \vartheta\right. \\
& \left.\times e^{i \gamma_{3}(z-d)} e^{i \rho \lambda \cos (\varphi-\vartheta)} d \vartheta\right) \lambda d \lambda=\frac{1}{(2 \pi)^{2}} \int_{0}^{+\infty} \lambda^{3} Q_{3 y}(\gamma) \\
& \times e^{i \gamma_{3}(z-d)}\left(\int_{0}^{2 \pi} \cos \vartheta \sin \vartheta e^{i \rho \lambda \cos (\varphi-\vartheta)} d \vartheta\right) d \lambda,
\end{aligned}
$$

где $\quad Q_{3 y}(\gamma)=\frac{1}{\omega \mu_{0} \mathfrak{D}(\lambda)}\left(a_{3 y} \cos ^{2}\left(\gamma_{2} d\right)+b_{3 y} \sin ^{2}\left(\gamma_{2} d\right)+\right.$ $\left.+i c_{3 y} \cos \left(\gamma_{2} d\right) \sin \left(\gamma_{2} d\right)\right)$.

Учитывая интегральное представление (56), получим

$$
\begin{aligned}
& E_{3, y}(\rho, \varphi, z)=\frac{\sin 2 \varphi}{4 \pi} \int_{0}^{+\infty} \lambda^{3} Q_{3 y}(\gamma) \\
& \times\left(J_{0}(\rho \lambda)-\frac{2}{\rho \lambda} J_{1}(\rho \lambda)\right) e^{i \gamma_{3}(z-d)} d \lambda .
\end{aligned}
$$

Наконец, для составляющей $E_{3, z}$, учитывая (65), получим аналогично

$$
\begin{aligned}
E_{3, z}(x, y, z)= & -\frac{1}{(2 \pi)^{2}} \int_{-\infty}^{+\infty} \int_{-\infty}^{+\infty}\left(\frac{\xi \hat{E}_{3, x}^{+}}{\gamma_{3}}+\frac{\eta \hat{E}_{3, y}^{+}}{\gamma_{3}}\right) \\
& \times e^{i \gamma_{3}(z-d)} e^{i(\xi x+\eta y)} d \xi d \eta .
\end{aligned}
$$

Переходя к полярным координатам, находим следующее выражение:

$$
\begin{aligned}
& E_{3, z}(\rho, \varphi, z)=-\frac{1}{(2 \pi)^{2}} \int_{0}^{+\infty} \frac{Q_{3 x}(\gamma) \lambda^{2} e^{i \gamma_{3}(z-d)}}{\gamma_{3}} \\
& \times\left(\int_{0}^{2 \pi} \cos \vartheta e^{i \rho \lambda \cos (\varphi-\vartheta)} d \vartheta\right) d \lambda \\
& -\frac{1}{(2 \pi)^{2}} \int_{0}^{+\infty} \frac{\left(R_{3 x}(\gamma)+Q_{3 y}(\gamma)\right) \lambda^{4}}{\gamma_{3}} e^{i \gamma_{3}(z-d)} \\
& \times\left(\int_{0}^{2 \pi} \cos \vartheta \sin ^{2} \vartheta e^{i \rho \lambda \cos (\varphi-\vartheta)} d \vartheta\right) d \lambda .
\end{aligned}
$$

Учитывая (68), (69), получим

$$
\begin{aligned}
& E_{3, z}(\rho, \varphi, z)=-\frac{i \cos \varphi}{2 \pi} \int_{0}^{+\infty} \frac{Q_{3 x}(\gamma) \lambda^{2} J_{1}(\rho \lambda)}{\gamma_{3}} e^{i \gamma_{3}(z-d)} d \lambda \\
& -\frac{i \cos \varphi \sin ^{2} \varphi}{2 \pi} \int_{0}^{+\infty} \frac{\left(R_{3 x}(\gamma)+Q_{3 y}(\gamma)\right) \lambda^{4} J_{1}(\rho \lambda)}{\gamma_{3}} e^{i \gamma_{3}(z-d)} d \lambda \\
& -\frac{i \cos \varphi\left(1-4 \sin ^{2} \varphi\right)}{2 \pi} \\
& \times \int_{0}^{+\infty} \frac{\left(R_{3 x}(\gamma)+Q_{3 y}(\gamma)\right) \lambda^{3} J_{2}(\rho \lambda)}{\rho \gamma_{3}} e^{i \gamma_{3}(z-d)} d \lambda .
\end{aligned}
$$

Таким образом, мы получили точные аналитические выражения для электрических полей в полупространствах, окружающих пленку. 


\section{Элементарный излучатель на свободной границе пленки золота в схеме Кречмана}

Данная работа задумывалась как продолжение работы по изучению явлений, связанных с поверхностной плазмонной волной, которая возбуждается по схеме Кречмана и распространяется вдоль свободной поверхности пленки металла (например, золота) определенной толщины, нанесенной на призму [18]. На свободной поверхности пленки возбуждается волна, которая имеет высокую интенсивность на границе раздела и экспоненциально спадает в направлении, нормальном к границе. Если поместить на свободной поверхности молекулу или наноструктуру, в которой будет индуцироваться поверхностной волной дипольный момент только параллельно границе, то мы получим рассмотренный выше элементарный излучатель, параллельный пленке. Интерес представляет пространственная направленность излучения этого диполя в двух случаях: при излучении диполя на границе двух полупространств без пленки и с металлической пленкой, по свободной границе которой может распространяться поверхностная плазмонная волна. Это сравнение поможет понять влияние поверхностной волны на направленные свойства элементарного излучателя.

Итак, выберем частоту излучения диполя $\omega$, соответствующую длине волны в вакууме, равной $\lambda_{0}=633 \mathrm{~nm}$, показатель преломления полупространства призмы на этой частоте выберем равным $n_{p}=1.6\left(\varepsilon_{1}=2.56 \varepsilon_{0}\right.$, где $\varepsilon_{0}-$ диэлектрическая проницаемость вакуума), показатель преломления свободного полупространства над пленкой $n_{f}=1\left(\varepsilon_{3}=\varepsilon_{0}\right)$. Диэлектрическую проницаемость золотой пленки на заданной частоте примем равной $\varepsilon_{2}=(-11.6+i 1.2) \varepsilon_{0} \quad[12]$, а ее толщину $d=48.6 \mathrm{~nm}$. Как было показано [18], при таких параметрах в пленке наилучшим образом возбуждается поверхностная плазмонная волна при падении плоской волны $p$-поляризации со стороны призмы под углом $\alpha_{\mathrm{opt}}=41.0^{\circ}$ к нормали. Для того чтобы оценить влияние такой пленки на излучение диполя в полупространство призмы и свободное полупространство, были вычислены диаграммы направленности в $H$ - и $E$-плоскостях диполя, т.е. в плоскостях, нормальных границе, проходящих через центр диполя, в его направлении и ему перпендикулярном (в плоскостях $Y Z$ и $X Z$ ) соответственно. Диаграммы вычислялись по амплитуде поля и нормировались на максимум излучения.

На рис. 6, $a$ показаны диаграммы направленности в $H$-плоскости, нормированные на максимум излучения. Рассматривается излучение в призму для двух случаев. Первый случай - пленка отсутствует (кривая 1), при этом диполь расположен на границе двух полупространств с диэлектрическими проницаемостями $\varepsilon_{1}$ и $\varepsilon_{3}$. Второй случай - с пленкой (кривая 2 ), когда диполь расположен на свободной границе золотой пленки
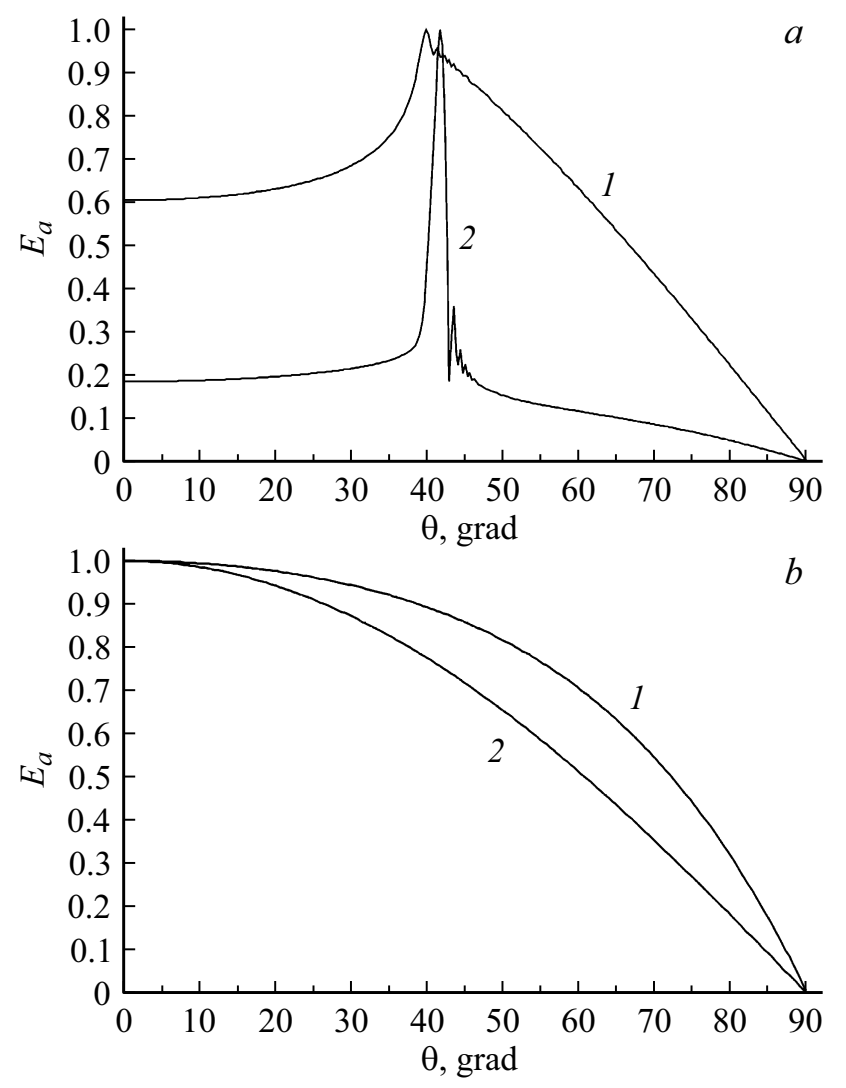

Рис. 6. Нормированные диаграммы направленности по амплитуде поля, вычисленные в $H$-плоскости, элементарного диполя, расположенного на границе двух полупространств (кривые 1) и на границе золотой пленки оптимальной толщины, нанесенной на призму (кривые 2 ), в сторону призмы $(a)$ и в сторону свободного пространства $(b)$.

(рис. 5) (на границе областей с проницаемостями $\varepsilon_{2}$ и $\varepsilon_{3}$ ). Диаграммы направленности представляют собой графики зависимости амплитуды электрического поля $E_{a}$ в точках на линии пересечения плоскости $Y Z$ и сферы большого радиуса $R_{S}$ от полярного угла $\theta$. Центр сферы находился в начале координат. Угол $\theta$ отсчитывался от оси $Z$. В численных расчетах радиус сферы принимался равным $R_{s}=790 \lambda_{0}$, т.е. поля вычислялись в дальней зоне излучения диполя.

Из рис. $6, a$ видно, что в отсутствие металлической пленки максимум излучения в призму примерно соответствует углу полного внутреннего отражения призмы (кривая 1), который равен $38.7^{\circ}$. Когда появляется металлическая пленка, возникает резкий пик излучения (кривая 2), однако нельзя связать этот пик на диаграмме с возбуждением поверхностной волны, так как она не должна давать вклад в диаграмму в $H$-плоскости из-за $p$-поляризации. Некоторые интерференционные биения на кривых, как показали расчеты, определяются конечностью радиуса $R_{s}$ и в пределе бесконечного радиуса исчезают.

На рис. $6, b$ представлены такие же диаграммы направленности в $H$-плоскости, но в сторону свободного 

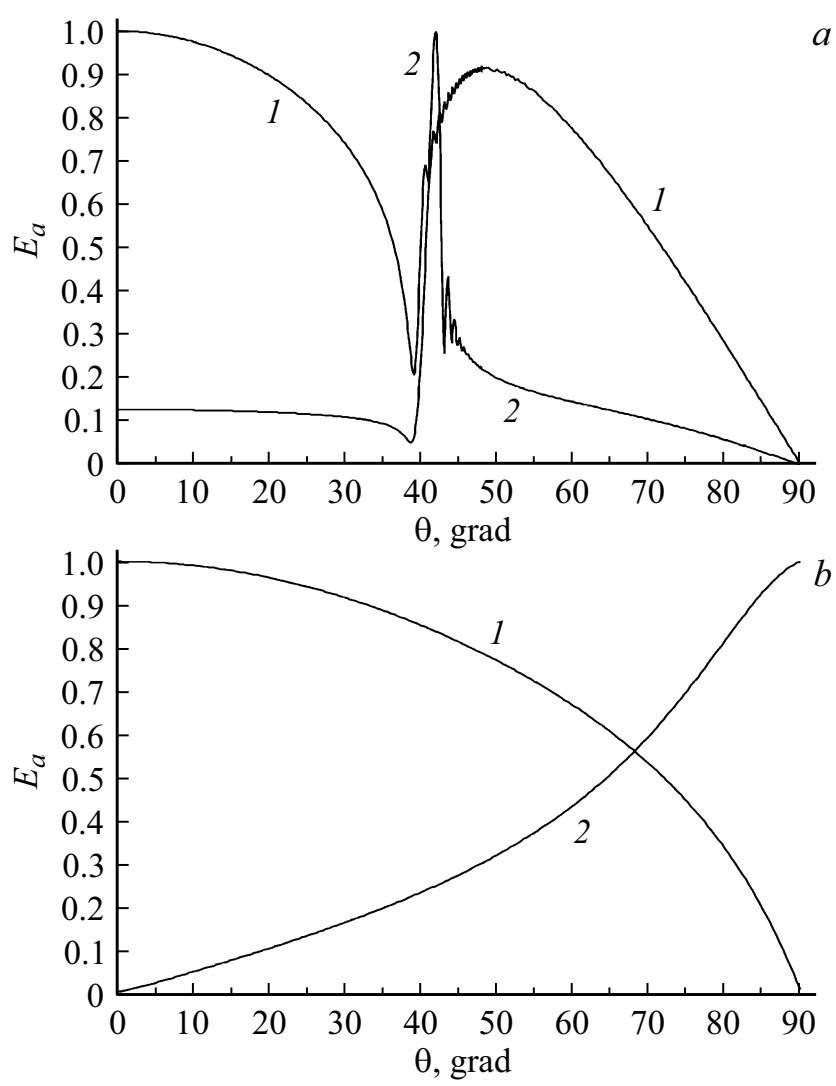

Рис. 7. Нормированные диаграммы направленности по амплитуде поля, вычисленные в $E$-плоскости, элементарного диполя, расположенного на границе двух полупространств (кривые 1 ) и на границе золотой пленки оптимальной толщины, нанесенной на призму (кривые 2), в сторону призмы $(a)$ и в сторону свободного пространства $(b)$.

полупространства (в область с проницаемостью $\varepsilon_{3}$ ). Из рисунка видно, что наличие металлической пленки качественно не меняет направленных свойств излучения диполя в $H$-плоскости в сторону свободного полупространства.

Далее представляет интерес, как влияет металлическая пленка на излучение в $E$-плоскости диполя в оба полупространства, так как поверхностная плазмонная волна на поверхности пленки имеет р-поляризацию и должна влиять на направленность излучения в $E$-плоскости. На рис. 7, $a$ показаны диаграммы направленности диполя в $E$-плоскости при излучении в призму. Первый случай - пленка отсутствует (кривая 1), при этом диполь расположен на границе двух полупространств с диэлектрическими проницаемостями $\varepsilon_{1}$ и $\varepsilon_{3}$. Второй случай - с пленкой (кривая 2), когда диполь расположен на свободной границе золотой пленки (рис. 5) (на границе областей с проницаемостями $\varepsilon_{2}$ и $\varepsilon_{3}$ ). На диаграмме (кривая 2, рис. $7, a$ ) виден резкий максимум излучения под углом, примерно равным углу полного внутреннего отражения. Зависимость качественно полностью аналогична диаграмме в $H$-плоскости (кривая 2, рис. $6, a)$.
Наконец, были вычислены диаграммы направленности в $E$-плоскости в сторону свободного пространства $\varepsilon_{3}$ (рис. $7, b)$. Появление металлической пленки существенно меняет диаграмму направленности диполя. Без пленки излучение преимущественно нормально к границе. С пленкой - волны излучаются преимущественно под большими углами, близкими к 90. Кажется логичным предположить, что такое изменение обусловлено возбуждающейся поверхностной плазмонной волной на свободной границе металлической пленки.

\section{Заключение}

Итак, предложено теоретическое рассмотрение электромагнитного излучения элементарного дипольного источника, расположенного в плоскослоистой среде. Предложен аналитический метод сведения электромагнитных полей к одномерным интегралам для задачи излучения точечного диполя, расположенного на границе пленки. Показана общность метода. Рассмотрена прикладная задача - нахождение направленных характеристик излучения нанообъектов, находящихся на свободной поверхности пленки золота оптимальной толщины в схеме Кречмана. Показано, что наличие металлической пленки, в которой может возбуждаться поверхностная волна, существенно меняет диаграмму направленности излучателя как в свободное полупространство, так и в полупространство призмы.

\section{Список литературы}

[1] Kretschmann E., Raether H.Z. // Naturforsch. 1968. V. A 23. P. 2135.

[2] Raether H. Surface Plasmons. Berlin: Springer-Verlag, 1988. $353 \mathrm{p}$.

[3] Barnes W.L. // J. Opt. A: Pure Appl. Opt. 2006. V. 8. P. S87.

[4] Garabedian R., Gonzalez C., Richards J. et al. // Sensors and Actuators. A. 1994. V. 43. P. 202.

[5] Петрин А.Б. // Опт. и спектр. 2018. Т. 125. № 6. С. 830.

[6] Sommerfeld A. // Ann. Physik. (Leipzig). 1926. V. 81. P. 1135.

[7] Wait J.R. // IEEE Antennas and Propagation Magazine. 1998. V. 40. N 5. P. 7.

[8] King R.W.P., Smith G.S. Antennas in Matter. Cambridge, MA: M.I.T. Press, 1981.

[9] Wu T.T. // J. Appl. Phys. 1957. V. 28. N 3. P. 299.

[10] Jansen R.H. // IEEE Trans. Microwave Theory Tech. 1985. V. 33. N 10. P. 1043.

[11] King R.W.P. // IEEE Trans. Microwave Theory Tech. 1988. V. 36. N 6. P. 1080.

[12] Новотный Л., Хехт Б. Основы нанооптики. Пер. с англ. / Под ред. Самарцева В.В. М.: Физматлит, 2009.

[13] Pendry J.B. // Phys. Rev. Lett. 2000. V. 85. N 18. P. 3966.

[14] Петрин А.Б. // ЖЭТФ. 2008. Т. 134. В. 3(9). С. 436.

[15] Петрин А.Б. // Опт. и спектр. 2018. Т. 125. № 3. С. 375.

[16] Петрин А.Б. // Опт. и спектр. 2019. Т. 126. № 3. С. 350.

[17] Петрин А.Б. // Опт. и спектр. 2019. Т. 127. № 4. С. 654.

[18] Петрин А.Б. // Опт. и спектр. 2019. Т. 127. № 6. С. 1051. 